\title{
Traditional Uses and Pharmacologically Active Constituents of Dendrobium Plants for Dermatological Disorders: A Review
}

\author{
Yue-Hu Wang ${ }^{1}$
}

Received: 5 March 2021 / Accepted: 8 April 2021 / Published online: 20 April 2021

(c) The Author(s) 2021

\begin{abstract}
Dendrobium Sw. is one of the largest genera in the orchidaceous family and includes 900-2000 species. Among them, more than 80 Dendrobium species have been reported in China. However, there are only six Dendrobium species, namely, $D$. bigibbum var. superbum (syn. D. phalaenopsis), D. chrysanthum, D. fimbriatum, D. loddigesii, D. nobile, and D. officinale (syn. D. candidum), listed in the New Inventory of Existing Cosmetic Ingredients in China Launched. Artificial planting of Dendrobium species has been a great success in China. To better utilize Dendrobium resources for medicinal and cosmetic purposes, we summarize their traditional uses and pharmacologically active compounds for treating dermatological disorders in this review. "Orchidaceae", "Dendrobium", "traditional use", "ethnobotany", "dermatological disorder", and "skin disease" were used as search terms to screen the literature. Cited references were collected between 1970 and 2020 from the Web of Science, China National Knowledge Internet (CNKI), SciFinder, Google Scholar, and Chinese books. From the search, it was found that there are 22 Dendrobium species with traditional uses in dermatological disorders, and 131 compounds from Dendrobium plants have been reported to possess anti-inflammatory, antimicrobial, antioxidant, antiaging, anti-psoriasis, and tyrosinase-inhibitory activities, implying that Dendrobium plants are important resources for the discovery of active compounds and the development of new drugs and cosmetics. D. crepidatum, D. denneanum, D. loddigesii, D. nobile, and $D$. officinale have been extensively studied. More research on other Dendrobium species is needed. The major active compounds found in Dendrobium species are phenanthrenes, alkaloids, flavonoids, phenylpropanoids, and lignans. Several compounds, such as loddigesiinol A, (S)-5-methoxy-2,4,7,9-tetrahydroxy-9,10-dihydrophenanthrene, $(S)$-4-methoxy-2,5,7,9tetrahydroxy-9,10-dihydrophenanthrene, 2,5-dihydroxy-4-methoxy-phenanthrene 2 - $O$ - $\beta$-D-glucopyranoside, $(9 R)-1,2,5,9$ tetrahydroxy-9,10-dihydrophenanthrene 5-O- $\beta$-D-glucopyranoside, (+)-homocrepidine A, and vicenin 2 , have significant anti-inflammatory activities and inhibit nitric oxide (NO) production with $\mathrm{IC}_{50}$ values less than $5 \mu \mathrm{M}$, and these compounds are worthy of further study.
\end{abstract}

\section{Graphic Abstract}

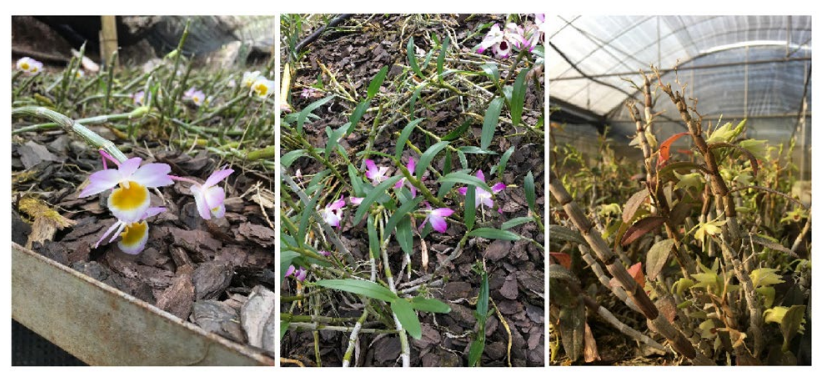

A planting base of Dendrobium crepidatum (left), D. nobile (middle), and D. officinale (right) in Yunnan, China

Keywords Orchidaceae $\cdot$ Dendrobium $\cdot$ Traditional uses $\cdot$ Dermatological disorders $\cdot$ Anti-inflammatory

Extended author information available on the last page of the article 


\section{Introduction}

Even though human skin is not the largest organ by weight or functional surface area [1], it is the main interface of the human body with the external environment. The skin meets the environment directly and thus is vulnerable to various types of damage. For this function, the skin possesses remarkable barrier qualities that protect humans from external pathogens and prevent the uncontrolled loss of water from the body. Although mortality rates for skin diseases are generally relatively low, they are often persistent, difficult to treat, can significantly impact quality of life and have a major psychological impact [2].

Dendrobium Sw. is one of the most important genera of Orchidaceae. The genus is one of the largest members of the orchidaceous family and includes 900-2000 species [3]. Dendrobium plants are mainly distributed in the tropics and subtropics in southern Asia, Oceanica, and elsewhere [4]. According to Plants of the World Online, there are 1,556 accepted Dendrobium species at present [5]. Among them, more than 80 Dendrobium species have been reported in China [4]. However, there are only six Dendrobium species, namely, D. bigibbum var. superbum Rchb.f. (syn. D. phalaenopsis Fitzg.), D. chrysanthum Wall. ex Lindl., D. fimbriatum Hook., D. loddigesii Rolfe, D. nobile Lindl, and D. officinale Kimura et Migo (syn. D. candidum Wall. ex Lindl.), listed in the New Inventory of Existing Cosmetic Ingredients in China Launched (IECIC 2015, Final Version) [6].

Artificial planting of Dendrobium species has been a great success in China. The total mass of annual cultivated Dendrobium plants in China now exceeds 19 million kg [7]. To better utilize Dendrobium resources for medicinal and cosmetic purposes, we summarize their traditional uses and pharmacologically active constituents for treating dermatological disorders. "Orchidaceae", "Dendrobium", "traditional use", "ethnobotany", "dermatological disorder", and "skin disease" were used as search terms to screen the literature. Cited references were collected between 1970 and 2020 from the Web of Science, China National Knowledge Internet (CNKI), SciFinder, Google Scholar, and Chinese books. For pharmacological activities, only extracts or compounds with $\mathrm{IC}_{50}, \mathrm{EC}_{50}$, or MIC values less than $100 \mu \mathrm{g} / \mathrm{mL}$ were cited.

\section{Traditional Uses of Dendrobium Species for Treating Dermatological Disorders}

Traditional uses of 22 Dendrobium species for treating dermatological disorders by local people in Australia, Bangladesh, China, India, Indonesia, Liberia, Malaysia, and Nepal are found in the literature (Table 1).

As shown in Table 1, the uses of Dendrobium plants include treatments of boils [Dendrobium alpestre Royle, D. crumenatum Sw., D. densiflorum Lindl., D. discolor Lindl., D. fimbriatum, D. monticola P.F. Hunt \& Summerh., and Dendrobium sp. (local name: gulubalama boblogie)], cuts (Dendrobium affine Steudel, D. fimbriatum, and D. nobile), burns [D. affine, D. amoenum Wall. ex Lindl., D. aphyllum (Roxb.) C.E.C. Fisch., D. nobile, and D. polyanthum Wall. ex Lindl.], eczema (D. macraei Lindl. and D. polyanthum), impetigo (D. denneanum Kerr), infected nails (Dendrobium purpureum Roxb.), itchy skin (Dendrobium affine Steudel), pimples (D. alpestre, D. crumenatum, D. densiflorum, D. fimbriatum, and $D$. monticola), scalds (D. aphyllum and D. polyanthum), skin allergies (D. macraei and D. macrostachyum Lindl.), sores (D. affine, D. canaliculatum R. Br., and D. chrysanthum), ringworm (D. discolor), ulcer (D. hancockii Rolfe), and wounds (D. amoenum, D. aphyllum, D. chrysanthum, D. fimbriatum, and D. nobile). The treatments of boils (seven species), burns (five species), pimples (five species), and wounds (five species) are the most common uses (Table 1).

Plant parts used of Dendrobium species for treating dermatological disorders include aerial parts (one species), bulbs (three species), leaves (eight species), pseudobulbs (six species), pseudostems (one species), roots (three species), seeds (one species), stems (seven species), and whole plants (two species). Leaves, pseudobulbs, and stems are the most common parts used.

\section{Pharmacological Activities of Extracts, Preparations, and Chemical Constituents from Dendrobium Plants for Treating Dermatological Disorders}

Some extracts, preparations, and chemical constituents from Dendrobium plants exhibit pharmacological activities related to dermatological disorders, such as antiinflammatory, antimicrobial, antioxidant, antiaging, antipsoriasis, hair growth promoting, skin-moisturizing, and tyrosinase-inhibitory activities. A part of pharmacological activities is related to the traditional uses of Dendrobium plants. For example, anti-inflammatory activities are associated with treatments of eczema, itchy skin, and skin 


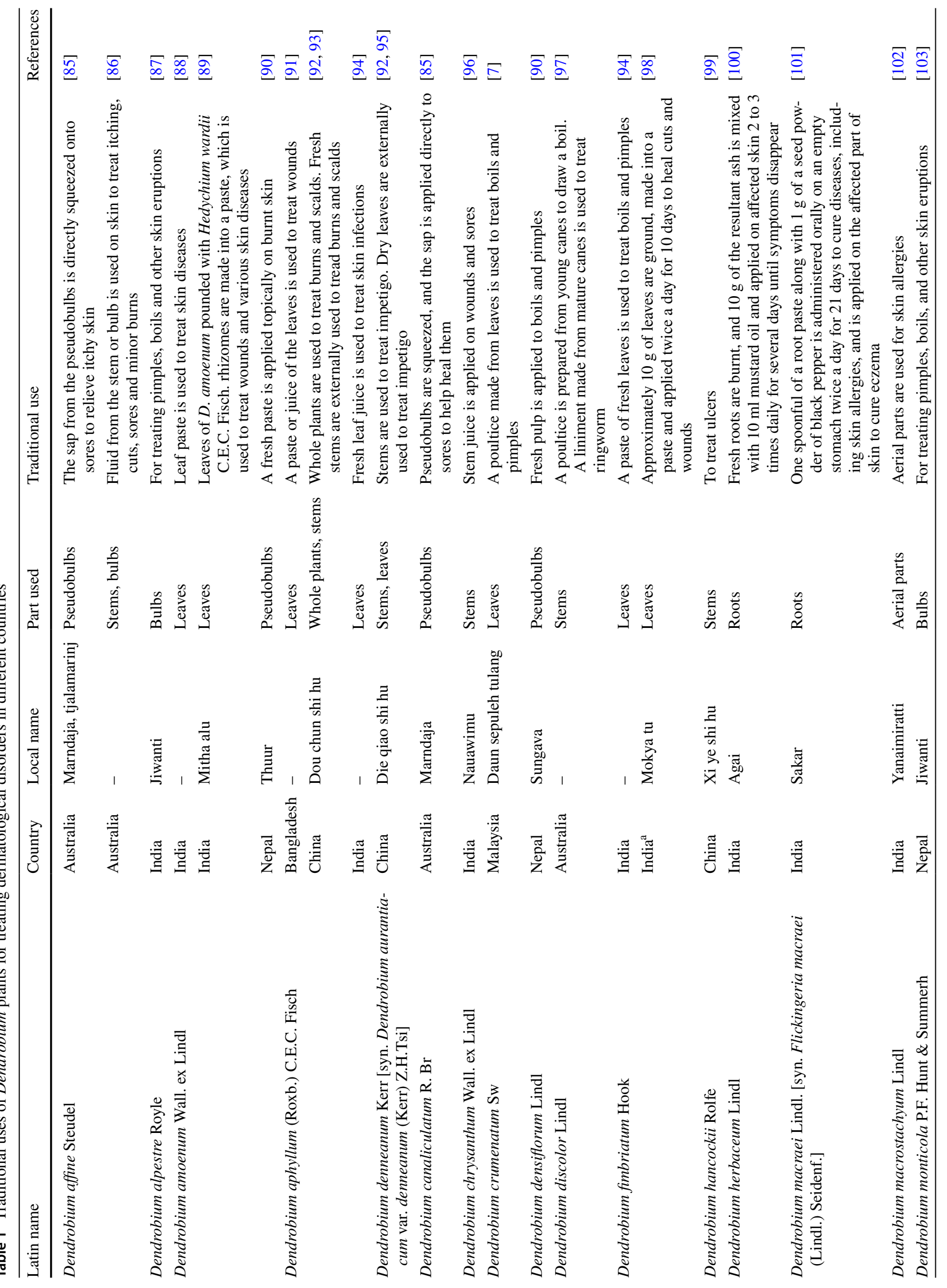




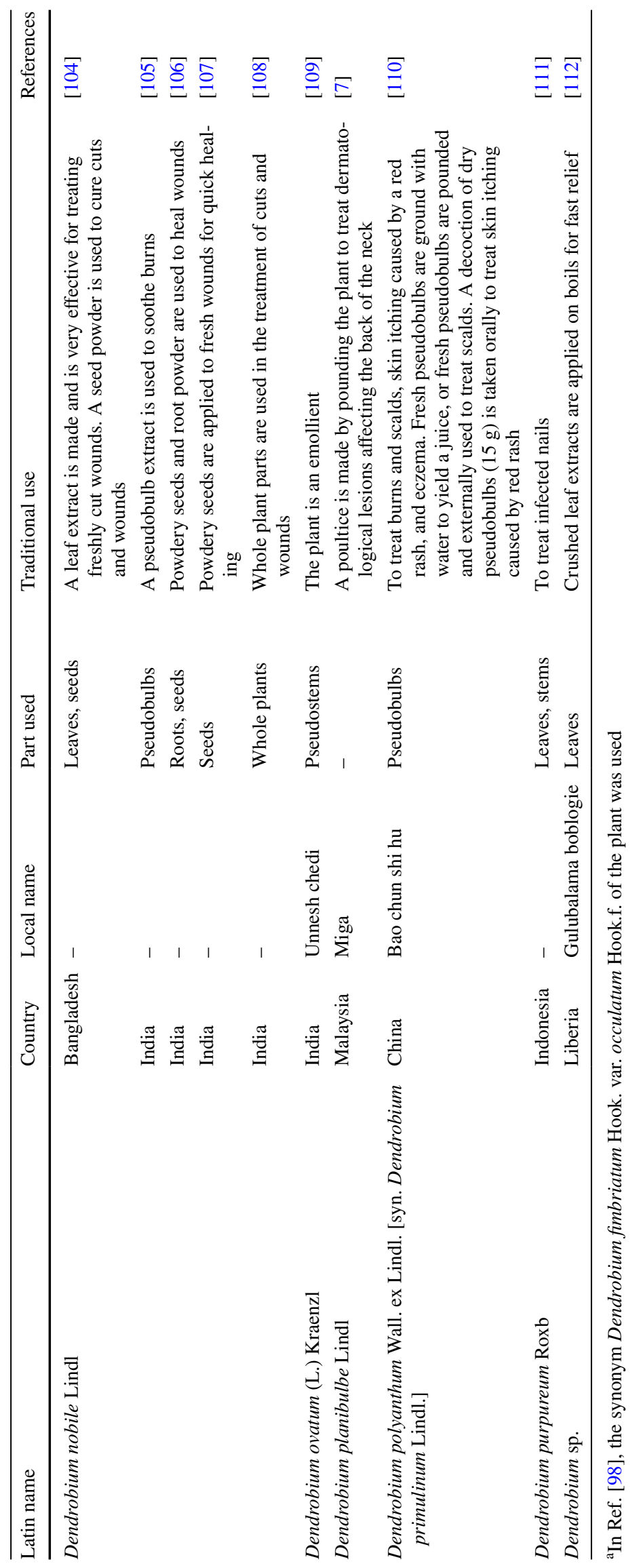


Table 2 Pharmacologically active compounds (1-131) from Dendrobium plants

\begin{tabular}{|c|c|c|c|c|c|}
\hline No. & Name & Type & Source & Pharmacological activities $^{\mathrm{a}}$ & References \\
\hline 1 & Batatasin III & Bibenzyls & D. loddigesii & $\begin{array}{l}\text { Antiaging (collagen production: } \\
\mathrm{EC}_{50} 3.2 \mu \mathrm{g} / \mathrm{mL} \text { ), anti-inflam- } \\
\left.\text { matory (NO: } \mathrm{IC}_{50} 21.9 \mu \mathrm{M}\right) \text {, and } \\
\text { antioxidant (DPPH: } \mathrm{IC}_{50} 50.1 \mu \mathrm{g} / \\
\mathrm{mL} \text { ) }\end{array}$ & {$[20,49]$} \\
\hline \multirow[t]{2}{*}{2} & \multirow[t]{2}{*}{ 3,3',5-Trihydroxybibenzyl } & \multirow[t]{2}{*}{ Bibenzyls } & \multirow[t]{2}{*}{ D. loddigesii } & $\begin{array}{l}\text { Anti-inflammatory (NO: } \mathrm{IC}_{50} \\
13.1 \mu \mathrm{M}) \text { and antioxidant (DPPH: } \\
85.8 \mu \mathrm{M})\end{array}$ & {$[20]$} \\
\hline & & & & $\begin{array}{l}\text { Tyrosinase-inhibitory }\left(\mathrm{IC}_{50} 37.9 \mu \mathrm{g} /\right. \\
\mathrm{mL} \text { ) }\end{array}$ & [49] \\
\hline 3 & 3,4'-Dihydroxy-5-methoxybibenzyl & Bibenzyls & D. officinale & Antioxidant (ABTS: $5.3 \mu \mathrm{M}$ ) & {$[66]$} \\
\hline 4 & 3-Hydroxy-4',5-dimethoxybibenzyl & Bibenzyls & D. heterocarpum & Anti-inflammatory & {$[15]$} \\
\hline 5 & $\begin{array}{l}\text { 4,4'-Dihydroxy-3,5- } \\
\text { dimethoxybibenzyl }\end{array}$ & Bibenzyls & D. loddigesii & $\begin{array}{l}\text { Anti-inflammatory (NO: } \mathrm{IC}_{50} \\
49.3 \mu \mathrm{M}) \text { and antioxidant (DPPH: } \\
\left.\mathrm{IC}_{50} 94.5 \mu \mathrm{M}\right)\end{array}$ & {$[20]$} \\
\hline 6 & $\begin{array}{l}\text { 3,3'-Dihydroxy-4,5- } \\
\text { dimethoxybibenzyl }\end{array}$ & Bibenzyls & D. williamsonii & Antioxidant (DPPH: $\mathrm{IC}_{50} 19.5 \mu \mathrm{M}$ ) & [74] \\
\hline \multirow[t]{5}{*}{7} & \multirow[t]{5}{*}{ Gigantol } & \multirow[t]{5}{*}{ Bibenzyls } & D. draconis & Antioxidant (DPPH: $\mathrm{IC}_{50} 17.7 \mu \mathrm{M}$ ) & [47] \\
\hline & & & D. heterocarpum & Anti-inflammatory & [15] \\
\hline & & & D. loddigesii & Antioxidant & [49] \\
\hline & & & D. nobile & $\begin{array}{l}\text { Anti-inflammatory (NO: } \mathrm{IC}_{50} \\
32.9 \mu \mathrm{M}) \text { and antioxidant (DPPH: } \\
\left.\mathrm{IC}_{50} 56.4 \mu \mathrm{M}\right)\end{array}$ & [23] \\
\hline & & & Dendrobium species & $\begin{array}{l}\text { Antibacterial (Staphylococcus } \\
\text { aureus: MIC } 82.2 \mu \mathrm{g} / \mathrm{mL} \text { ) }\end{array}$ & {$[32,33]$} \\
\hline \multirow[t]{2}{*}{8} & \multirow[t]{2}{*}{ Tristin } & \multirow[t]{2}{*}{ Bibenzyls } & D. loddigesii & Antioxidant & [49] \\
\hline & & & D. officinale & $\begin{array}{l}\text { Antioxidant (ABTS: } \mathrm{IC}_{50} 9.0 \mu \mathrm{M} \text {; } \\
\text { DPPH: } \mathrm{IC}_{50} 34.5 \mu \mathrm{M} \text { ) }\end{array}$ & {$[66]$} \\
\hline \multirow[t]{4}{*}{9} & \multirow[t]{4}{*}{ Moscatilin } & \multirow[t]{4}{*}{ Bibenzyls } & D. loddigesii & Antioxidant & [49] \\
\hline & & & D. nobile & $\begin{array}{l}\text { Anti-inflammatory (NO: } \mathrm{IC}_{50} 27.6 \\
\text { and } 36.8 \mu \mathrm{M} \text { ) and antioxidant } \\
\left.\text { (DPPH: } \mathrm{IC}_{50} 14.5 \mu \mathrm{M}\right)\end{array}$ & {$[22,23]$} \\
\hline & & & D. secundum & Antioxidant (DPPH: $\mathrm{IC}_{50} 5.1 \mu \mathrm{M}$ ) & {$[70]$} \\
\hline & & & D. williamsonii & Antioxidant (DPPH: $\mathrm{IC}_{50} 8.5 \mu \mathrm{M}$ ) & [74] \\
\hline 10 & Dendrobin A & Bibenzyls & D. nobile & Antioxidant (DPPH: $\mathrm{IC}_{50} 40.3 \mu \mathrm{M}$ ) & [23] \\
\hline 11 & Chrysotoxine & Bibenzyls & D. nobile & Antioxidant (DPPH: $\mathrm{IC}_{50} 14.0 \mu \mathrm{M}$ ) & {$[23]$} \\
\hline 12 & Dendrocandin E & Bibenzyls & D. officinale & Antioxidant (DPPH: $\mathrm{IC}_{50} 15.6 \mu \mathrm{M}$ ) & {$[61]$} \\
\hline \multirow[t]{2}{*}{13} & \multirow{2}{*}{$\begin{array}{l}\text { 4,5,4'-Trihydroxy-3,3'- } \\
\text { dimethoxybibenzyl }\end{array}$} & \multirow[t]{2}{*}{ Bibenzyls } & D. loddigesii & Antioxidant & [49] \\
\hline & & & D. secundum & Antioxidant (DPPH: $\mathrm{IC}_{50} 15.9 \mu \mathrm{M}$ ) & [70] \\
\hline \multirow[t]{2}{*}{14} & \multirow[t]{2}{*}{ Erianin } & \multirow[t]{2}{*}{ Bibenzyls } & \multirow[t]{2}{*}{ D. chrysotoxum } & $\begin{array}{l}\text { Antibacterial (srtA: } \mathrm{IC}_{50} 20.9 \mu \mathrm{g} / \\
\mathrm{mL} \text { ) }\end{array}$ & {$[34,35]$} \\
\hline & & & & Anti-psoriasis & {$[75]$} \\
\hline \multirow[t]{2}{*}{15} & \multirow[t]{2}{*}{ Crepidatin } & \multirow[t]{2}{*}{ Bibenzyls } & D. loddigesii & Antioxidant & [49] \\
\hline & & & D. nobile & Antioxidant (DPPH: $\mathrm{IC}_{50} 21.8 \mu \mathrm{M}$ ) & [23] \\
\hline 16 & Chrysotobibenzyl & Bibenzyls & D. nobile & $\begin{array}{l}\text { Anti-inflammatory (NO: } \mathrm{IC}_{50} \\
48.2 \mu \mathrm{M})\end{array}$ & {$[23]$} \\
\hline 17 & Aphyllone B & Bibenzyls & D. aphyllum & Antioxidant & [42] \\
\hline 18 & Dendrocandin C & Bibenzyls & D. officinale & Antioxidant (DPPH: $\mathrm{IC}_{50} 34.2 \mu \mathrm{M}$ ) & {$[61]$} \\
\hline 19 & Dendrocandin D & Bibenzyls & D. officinale & Antioxidant (DPPH: $\mathrm{IC}_{50} 34.5 \mu \mathrm{M}$ ) & {$[61]$} \\
\hline 20 & $\begin{array}{l}(S)-3,4, \alpha \text {-trihydroxy-5,4'- } \\
\text { dimethoxybibenzyl }\end{array}$ & Bibenzyls & D. officinale & Antioxidant (DPPH: $\mathrm{IC}_{50} 32.3 \mu \mathrm{M}$ ) & {$[64]$} \\
\hline 21 & Nobilin D & Bibenzyls & D. nobile & $\begin{array}{l}\text { Anti-inflammatory (NO: } \mathrm{IC}_{50} \\
15.3 \mu \mathrm{M}) \text { and antioxidant (DPPH: } \\
\left.\mathrm{IC}_{50} 19.9 \mu \mathrm{M}\right)\end{array}$ & {$[23]$} \\
\hline
\end{tabular}


Table 2 (continued)

\begin{tabular}{|c|c|c|c|c|c|}
\hline No. & Name & Type & Source & Pharmacological activities $^{\mathrm{a}}$ & References \\
\hline 22 & Nobilin A & Bibenzyls & D. nobile & Antioxidant (DPPH: $\mathrm{IC}_{50} 87.1 \mu \mathrm{M}$ ) & {$[56]$} \\
\hline 23 & Nobilin B & Bibenzyls & D. nobile & Antioxidant (DPPH: $\mathrm{IC}_{50} 32.2 \mu \mathrm{M}$ ) & {$[56]$} \\
\hline 24 & Nobilin C & Bibenzyls & D. nobile & $\begin{array}{l}\text { Antioxidant (DPPH: } \mathrm{IC}_{50} 47.4 \mu \mathrm{g} / \\
\mathrm{mL} \text { ) }\end{array}$ & {$[56]$} \\
\hline 25 & Loddigesiinol C & Bibenzyls & D. loddigesii & Antioxidant (DPPH: $\mathrm{IC}_{50} 23.7 \mu \mathrm{M}$ ) & [19] \\
\hline 26 & Loddigesiinol D & Bibenzyls & D. loddigesii & $\begin{array}{l}\text { Anti-inflammatory (NO: } \mathrm{IC}_{50} \\
69.7 \mu \mathrm{M})\end{array}$ & [19] \\
\hline 27 & Crepidatuol B & Bibenzyls & D. loddigesii & Antioxidant & {$[50]$} \\
\hline 28 & Trigonopol B & Bibenzyls & D. loddigesii & $\begin{array}{l}\text { Anti-inflammatory (NO: } \mathrm{IC}_{50} \\
26.3 \mu \mathrm{M} \text { ) and antioxidant (DPPH: } \\
\left.\mathrm{IC}_{50} 60.1 \mu \mathrm{M}\right)\end{array}$ & {$[20]$} \\
\hline 29 & Dendrocandin F & Bibenzyls & D. officinale & Antioxidant (DPPH: $\mathrm{IC}_{50} 55.8 \mu \mathrm{M}$ ) & {$[62]$} \\
\hline 30 & Dendrocandin G & Bibenzyls & D. officinale & Antioxidant (DPPH: $\mathrm{IC}_{50} 32.4 \mu \mathrm{M}$ ) & {$[62]$} \\
\hline 31 & Dendrocandin J & Bibenzyls & D. officinale & Aantioxidant (DPPH: $\mathrm{IC}_{50} 36.8 \mu \mathrm{M}$ ) & {$[63]$} \\
\hline 32 & Dendrocandin K & Bibenzyls & D. officinale & Antioxidant (DPPH: $\left.\mathrm{IC}_{50} 70.2 \mu \mathrm{M}\right)$ & {$[63]$} \\
\hline 33 & Nobilin E & Bibenzyls & D. nobile & $\begin{array}{l}\text { Anti-inflammatory (NO: } \mathrm{IC}_{50} \\
19.2 \mu \mathrm{M}) \text { and antioxidant (DPPH: } \\
\left.\mathrm{IC}_{50} 21.0 \mu \mathrm{M}\right)\end{array}$ & {$[23]$} \\
\hline 34 & Dendrocandin $\mathrm{H}$ & Bibenzyls & D. officinale & Antioxidant (DPPH: $\mathrm{IC}_{50} 32.4 \mu \mathrm{M}$ ) & {$[62]$} \\
\hline 35 & Dendrocandin L & Bibenzyls & D. officinale & Antioxidant (DPPH: $\mathrm{IC}_{50} 45.0 \mu \mathrm{M}$ ) & {$[63]$} \\
\hline 36 & (-)-Dendroparishiol & Bibenzyls & D. parishii & Anti-inflammatory & {$[28]$} \\
\hline 37 & Dendrocandin M & Bibenzyls & D. officinale & Antioxidant (DPPH: $\mathrm{IC}_{50} 60.5 \mu \mathrm{M}$ ) & {$[63]$} \\
\hline 38 & $\begin{array}{l}6 " \text {-De- } O \text {-methyldendrofindlaphenol } \\
\text { A }\end{array}$ & Bibenzyls & D. findlayanum & $\begin{array}{l}\text { Anti-inflammatory (NO: } \mathrm{IC}_{50} \\
21.4 \mu \mathrm{M})\end{array}$ & {$[14]$} \\
\hline \multirow[t]{2}{*}{39} & Dendrocandin I & Bibenzyls & D. heterocarpum & Anti-inflammatory & {$[15]$} \\
\hline & & & D. officinale & Antioxidant (DPPH: $\mathrm{IC}_{50} 21.3 \mu \mathrm{M}$ ) & {$[62]$} \\
\hline 40 & Dendrocandin P & Bibenzyls & D. officinale & Antioxidant (DPPH: $\mathrm{IC}_{50} 22.3 \mu \mathrm{M}$ ) & {$[63]$} \\
\hline 41 & Dendrocandin Q & Bibenzyls & D. officinale & Antioxidant (DPPH: $\mathrm{IC}_{50} 30.3 \mu \mathrm{M}$ ) & {$[63]$} \\
\hline 42 & Dendrocandin N & Bibenzyls & D. officinale & Antioxidant (DPPH: $\mathrm{IC}_{50} 87.6 \mu \mathrm{M}$ ) & {$[63]$} \\
\hline 43 & Dendrocandin $\mathrm{O}$ & Bibenzyls & D. officinale & Antioxidant (DPPH: $\mathrm{IC}_{50} 50.4 \mu \mathrm{M}$ ) & {$[63]$} \\
\hline 44 & Dendrocandin U & Bibenzyls & D. officinale & Antioxidant (ABTS: $\mathrm{IC}_{50} 10.0 \mu \mathrm{M}$ ) & {$[66]$} \\
\hline \multirow[t]{2}{*}{45} & Moscatin (plicatol B) & Phenanthrenes & D. denneanum & $\begin{array}{l}\text { Anti-inflammatory (NO: } \mathrm{IC}_{50} \\
6.3 \mu \mathrm{M})\end{array}$ & {$[13]$} \\
\hline & & & D. loddigesii & $\begin{array}{l}\text { Anti-inflammatory (NO: } \mathrm{IC}_{50} \\
6.4 \mu \mathrm{M}) \text { and antioxidant (DPPH: } \\
\left.\mathrm{IC}_{50} 59.8 \mu \mathrm{M}\right)\end{array}$ & {$[19,50]$} \\
\hline 46 & $\begin{array}{l}\text { 5-Hydroxy-2,4-dimethoxyphenan- } \\
\text { threne }\end{array}$ & Phenanthrenes & D. loddigesii & $\begin{array}{l}\text { Anti-inflammatory (NO: } \mathrm{IC}_{50} \\
5.3 \mu \mathrm{M} \text { ) }\end{array}$ & [19] \\
\hline 47 & Confusarin & Phenanthrenes & D. nobile & Antioxidant (DPPH: $\mathrm{IC}_{50} 12.9 \mu \mathrm{M}$ ) & [57] \\
\hline 48 & Fimbriol B & Phenanthrenes & D. nobile & $\begin{array}{l}\text { Anti-inflammatory (NO: } \mathrm{IC}_{50} \\
28.9 \mu \mathrm{M})\end{array}$ & {$[22]$} \\
\hline 49 & Flavanthrinin & Phenanthrenes & D. nobile & Antioxidant (DPPH: $\mathrm{IC}_{50} 35.7 \mu \mathrm{M}$ ) & [57] \\
\hline \multirow[t]{2}{*}{50} & $\begin{array}{l}\text { 5,7-Dimethoxyphenanthrene- } \\
\text { 2,6-diol }\end{array}$ & Phenanthrenes & D. nobile & $\begin{array}{l}\text { Anti-inflammatory (NO: } \mathrm{IC}_{50} \\
37.7 \mu \mathrm{M})\end{array}$ & {$[22]$} \\
\hline & & & & Antioxidant (DPPH: $\mathrm{IC}_{50} 29.7 \mu \mathrm{M}$ ) & {$[57]$} \\
\hline 51 & $\begin{array}{l}\text { 3,4,8-Trimethoxyphenanthrene- } \\
\text { 2,5-diol }\end{array}$ & Phenanthrenes & D. nobile & $\begin{array}{l}\text { Anti-inflammatory (NO: } \mathrm{IC}_{50} \\
20.4 \mu \mathrm{M} \text { ) }\end{array}$ & {$[22]$} \\
\hline 52 & Loddigesiinol A & Phenanthrenes & D. loddigesii & $\begin{array}{l}\text { Anti-inflammatory (NO: } \mathrm{IC}_{50} \\
2.6 \mu \mathrm{M} \text { ) and antioxidant (DPPH: } \\
\left.\mathrm{IC}_{50} 26.1 \mu \mathrm{M}\right)\end{array}$ & [19] \\
\hline 53 & $\begin{array}{l}\text { 2,5-Dihydroxy-4,9-dimethoxyphen- } \\
\text { anthrene }\end{array}$ & Phenanthrenes & D. nobile & Antioxidant (DPPH: $\mathrm{IC}_{50} 34.8 \mu \mathrm{M}$ ) & {$[57]$} \\
\hline
\end{tabular}


Table 2 (continued)

\begin{tabular}{|c|c|c|c|c|c|}
\hline No. & Name & Type & Source & Pharmacological activities $^{\mathrm{a}}$ & References \\
\hline \multirow[t]{2}{*}{54} & Lusianthridin & Phenanthrenes & D. loddigesii & $\begin{array}{l}\text { Anti-inflammatory (NO: } \mathrm{IC}_{50} \\
4.6 \mu \mathrm{M}) \text { and antioxidant }\left(\mathrm{IC}_{50}\right. \\
62.2 \mu \mathrm{M})\end{array}$ & [19] \\
\hline & & & D. nobile & $\begin{array}{l}\text { Anti-inflammatory (NO: } \mathrm{IC}_{50} \\
9.6 \mu \mathrm{M})\end{array}$ & {$[22]$} \\
\hline \multirow[t]{3}{*}{55} & Hircinol & Phenanthrenes & D. draconis & Antioxidant (DPPH: $\mathrm{IC}_{50} 22.3 \mu \mathrm{M}$ ) & {$[47]$} \\
\hline & & & D. loddigesii & $\begin{array}{l}\text { Anti-inflammatory (NO: } \mathrm{IC}_{50} \\
29.2 \mu \mathrm{M})\end{array}$ & [19] \\
\hline & & & D. nobile & $\begin{array}{l}\text { Anti-inflammatory (NO: } \mathrm{IC}_{50} \\
26.4 \mu \mathrm{M} \text { ) }\end{array}$ & {$[22]$} \\
\hline \multirow[t]{2}{*}{56} & $\begin{array}{l}\text { 9,10-Dihydrophenanthrene-2,4,7- } \\
\text { triol }\end{array}$ & Phenanthrenes & D. denneanum & $\begin{array}{l}\text { Anti-inflammatory (NO: } \mathrm{IC}_{50} \\
32.7 \mu \mathrm{M})\end{array}$ & [13] \\
\hline & & & D. loddigesii & $\begin{array}{l}\text { Anti-inflammatory (NO: } \mathrm{IC}_{50} \\
8.6 \mu \mathrm{M}) \text { and antioxidant (DPPH: } \\
\left.\mathrm{IC}_{50} 14.1 \mu \mathrm{M}\right)\end{array}$ & {$[20,50]$} \\
\hline 57 & $\begin{array}{l}\text { 2-Methoxy-9,10-dihydrophenan- } \\
\text { threne-4,5-diol }\end{array}$ & Phenanthrenes & D. denneanum & $\begin{array}{l}\text { Anti-inflammatory (NO: } \mathrm{IC}_{50} \\
7.6 \mu \mathrm{M})\end{array}$ & [13] \\
\hline 58 & Coelonin & Phenanthrenes & D. nobile & $\begin{array}{l}\text { Anti-inflammatory (NO: } \mathrm{IC}_{50} \\
10.2 \mu \mathrm{M} \text { ) }\end{array}$ & {$[22]$} \\
\hline 59 & $\begin{array}{l}\text { 7-Methoxy-9,10-dihydrophenan- } \\
\text { threne-2,4,5-triol }\end{array}$ & Phenanthrenes & D. draconis & Antioxidant (DPPH: $\mathrm{IC}_{50} 10.2 \mu \mathrm{M}$ ) & {$[47]$} \\
\hline 60 & Erianthridin & Phenanthrenes & D. nobile & $\begin{array}{l}\text { Anti-inflammatory (NO: } \mathrm{IC}_{50} \\
19.5 \mu \mathrm{M})\end{array}$ & {$[22]$} \\
\hline 61 & Flavanthridin & Phenanthrenes & D. nobile & $\begin{array}{l}\text { Anti-inflammatory (NO: } \mathrm{IC}_{50} \\
34.1 \mu \mathrm{M})\end{array}$ & {$[22]$} \\
\hline 62 & Epheneranthol C & Phenanthrenes & D. nobile & $\begin{array}{l}\text { Anti-inflammatory (NO: } \mathrm{IC}_{50} \\
17.6 \mu \mathrm{M} \text { ) }\end{array}$ & {$[22]$} \\
\hline 63 & Ephemeranthol A & Phenanthrenes & D. nobile & $\begin{array}{l}\text { Anti-inflammatory (NO: } \mathrm{IC}_{50} \\
12.0 \mu \mathrm{M})\end{array}$ & {$[22]$} \\
\hline 64 & Rotundatin (plicatol C) & Phenanthrenes & D. loddigesii & $\begin{array}{l}\text { Anti-inflammatory (NO: } \mathrm{IC}_{50} \\
29.1 \mu \mathrm{M} \text { ) }\end{array}$ & [19] \\
\hline 65 & $\begin{array}{l}(S) \text {-4-Methoxy-9,10-dihydrophenan- } \\
\text { threne-2,5,9-triol }\end{array}$ & Phenanthrenes & D. denneanum & $\begin{array}{l}\text { Anti-inflammatory (NO: } \mathrm{IC}_{50} \\
27.4 \mu \mathrm{M} \text { ) }\end{array}$ & {$[13]$} \\
\hline 66 & $\begin{array}{l}\text { (S)-5-Methoxy-2,4,7,9-tetrahydroxy- } \\
\text { 9,10-dihydrophenanthrene }\end{array}$ & Phenanthrenes & D. denneanum & $\begin{array}{l}\text { Anti-inflammatory (NO: } \mathrm{IC}_{50} \\
3.1 \mu \mathrm{M} \text { ) }\end{array}$ & [13] \\
\hline 67 & $\begin{array}{l}\text { (S)-4-Methoxy-2,5,7,9-tetrahydroxy- } \\
\text { 9,10-dihydrophenanthrene }\end{array}$ & Phenanthrenes & D. denneanum & $\begin{array}{l}\text { Anti-inflammatory (NO: } \mathrm{IC}_{50} \\
4.2 \mu \mathrm{M} \text { ) }\end{array}$ & [13] \\
\hline 68 & Denbinobin & Phenanthrenes & D. moniliforme & Anti-inflammatory & {$[21]$} \\
\hline 69 & $\begin{array}{l}\text { 5-Methoxy-7-hydroxy-9,10-dihydro- } \\
\text { 1,4-phenanthrenequinone }\end{array}$ & Phenanthrenes & D. draconis & $\begin{array}{l}\text { Antioxidant (DPPH: } \mathrm{IC}_{50} 72.6 \mu \mathrm{g} / \\
\mathrm{mL} \text { ) }\end{array}$ & [47] \\
\hline 70 & Fimbriatone & Phenanthrenes & D. nobile & $\begin{array}{l}\text { Antioxidant (DPPH: } \mathrm{IC}_{50} 40.8 \mu \mathrm{g} / \\
\mathrm{mL} \text { ) }\end{array}$ & {$[57]$} \\
\hline 71 & Loddigesiinol B & Phenanthrenes & D. loddigesii & $\begin{array}{l}\text { Anti-inflammatory (NO: } \mathrm{IC}_{50} 9.9 \\
\text { and } 10.9 \mu \mathrm{M})\end{array}$ & {$[19,20]$} \\
\hline 72 & Loddigesiinol I & phenanthrenes & D. loddigesii & $\begin{array}{l}\text { Anti-inflammatory (NO: } \mathrm{IC}_{50} \\
7.5 \mu \mathrm{M})\end{array}$ & {$[20]$} \\
\hline 73 & Loddigesiinol J & Phenanthrenes & D. loddigesii & $\begin{array}{l}\text { Anti-inflammatory (NO: } \mathrm{IC}_{50} \\
14.6 \mu \mathrm{M})\end{array}$ & {$[20]$} \\
\hline 74 & Chrysotoxol A & Phenanthrenes & D. loddigesii & $\begin{array}{l}\text { Anti-inflammatory (NO: } \mathrm{IC}_{50} \\
10.9 \mu \mathrm{M} \text { ) and antioxidant (DPPH: } \\
\mathrm{IC}_{50} 23.2 \mu \mathrm{M} \text { ) }\end{array}$ & {$[20]$} \\
\hline 75 & Dendrochrysanene & Phenanthrenes & D. chrysanthum & Anti-inflammatory & [9] \\
\hline 76 & $\begin{array}{l}\text { 2,5-Dihydroxy-4-methoxy-phenan- } \\
\text { threne } 2-O-\beta \text {-D-glucopyranoside }\end{array}$ & Phenanthrenes & D. denneanum & $\begin{array}{l}\text { Anti-inflammatory (NO: } \mathrm{IC}_{50} \\
4.6 \mu \mathrm{M})\end{array}$ & [13] \\
\hline
\end{tabular}


Table 2 (continued)

\begin{tabular}{|c|c|c|c|c|c|}
\hline No. & Name & Type & Source & Pharmacological activities ${ }^{\mathrm{a}}$ & References \\
\hline 77 & $\begin{array}{l}\text { 2,5-Dihydroxy-4-methoxy- } \\
\text { phenanthrene } 2-O-\beta \text {-D- } \\
\text { apiofuranosyl- }(1 \rightarrow 6)-\beta \text {-D- } \\
\text { glucopyranoside }\end{array}$ & Phenanthrenes & D. denneanum & $\begin{array}{l}\text { Anti-inflammatory (NO: } \mathrm{IC}_{50} \\
16.9 \mu \mathrm{M})\end{array}$ & [13] \\
\hline 78 & $\begin{array}{l}\text { 2,5-Dihydroxy-4-methoxy- } \\
\text { phenanthrene } 2-O-\alpha \text {-L- } \\
\text { rhamnopyranosyl- }(1 \rightarrow 6)-\beta \text {-D- } \\
\text { glucopyranoside }\end{array}$ & Phenanthrenes & D. denneanum & $\begin{array}{l}\text { Anti-inflammatory (NO: } \mathrm{IC}_{50} \\
41.5 \mu \mathrm{M})\end{array}$ & [13] \\
\hline 79 & $\begin{array}{l}\text { (9R)-1,2,5,9-Tetrahydroxy-9,10-di- } \\
\text { hydrophenanthrene 5- } O-\beta \text {-D- } \\
\text { glucopyranoside }\end{array}$ & Phenanthrenes & D. denneanum & $\begin{array}{l}\text { Anti-inflammatory (NO: } \mathrm{IC}_{50} \\
0.7 \mu \mathrm{M})\end{array}$ & [13] \\
\hline 80 & Shihunine & Alkaloids & D. loddigesii & $\begin{array}{l}\text { Anti-inflammatory (NO: } \mathrm{IC}_{50} \\
11.5 \mu \mathrm{g} / \mathrm{mL} \text { ) }\end{array}$ & [18] \\
\hline 81 & Anosmine & Alkaloids & D. nobile & $\begin{array}{l}\text { Anti-inflammatory (NO: } \mathrm{IC}_{50} \\
16.1 \mu \mathrm{g} / \mathrm{mL} \text { ) }\end{array}$ & [18] \\
\hline 82 & (+)-Homocrepidine A & Alkaloids & D. crepidatum & $\begin{array}{l}\text { Anti-inflammatory (NO: } \mathrm{IC}_{50} \\
3.6 \mu \mathrm{M} \text { ) }\end{array}$ & [11] \\
\hline 83 & (-)-Homocrepidine A & Alkaloids & D. crepidatum & $\begin{array}{l}\text { Anti-inflammatory (NO: } \mathrm{IC}_{50} \\
22.8 \mu \mathrm{M} \text { ) }\end{array}$ & [11] \\
\hline 84 & Homocrepidine B & Alkaloids & D. crepidatum & $\begin{array}{l}\text { Anti-inflammatory (NO: } \mathrm{IC}_{50} \\
27.6 \mu \mathrm{M} \text { ) }\end{array}$ & [11] \\
\hline 85 & (+)-Dendrocrepidamine A & Alkaloids & D. crepidatum & $\begin{array}{l}\text { Anti-inflammatory (NO: } \mathrm{IC}_{50} \\
16.1 \mu \mathrm{M})\end{array}$ & {$[12]$} \\
\hline 86 & Dendrocrepidamine B & Alkaloids & D. crepidatum & $\begin{array}{l}\text { Anti-inflammatory (NO: } \mathrm{IC}_{50} \\
14.3 \mu \mathrm{M})\end{array}$ & [12] \\
\hline 87 & (-)-Crepidine & Alkaloids & D. crepidatum & $\begin{array}{l}\text { Anti-inflammatory (NO: } \mathrm{IC}_{50} \\
29.9 \mu \mathrm{M})\end{array}$ & [12] \\
\hline 88 & $(+)$-Crepidine & Alkaloids & D. crepidatum & $\begin{array}{l}\text { Anti-inflammatory (NO: } \mathrm{IC}_{50} \\
81.9 \mu \mathrm{M})\end{array}$ & [12] \\
\hline 89 & (-)-Dendrocrepidine A & Alkaloids & D. crepidatum & $\begin{array}{l}\text { Anti-inflammatory (NO: } \mathrm{IC}_{50} \\
18.5 \mu \mathrm{M})\end{array}$ & {$[12]$} \\
\hline 90 & (+)-Dendrocrepidine A & Alkaloids & D. crepidatum & $\begin{array}{l}\text { Anti-inflammatory (NO: } \mathrm{IC}_{50} \\
30.2 \mu \mathrm{M})\end{array}$ & [12] \\
\hline 91 & (-)-Isocrepidamine & Alkaloids & D. crepidatum & $\begin{array}{l}\text { Anti-inflammatory (NO: } \mathrm{IC}_{50} \\
16.3 \mu \mathrm{M})\end{array}$ & [12] \\
\hline 92 & $(+)$-Isocrepidamine & Alkaloids & D. crepidatum & $\begin{array}{l}\text { Anti-inflammatory (NO: } \mathrm{IC}_{50} \\
73.0 \mu \mathrm{M})\end{array}$ & [12] \\
\hline 93 & Dendrocrepidine B & Alkaloids & D. crepidatum & $\begin{array}{l}\text { Anti-inflammatory (NO: } \mathrm{IC}_{50} \\
51.8 \mu \mathrm{M})\end{array}$ & [10] \\
\hline 94 & Dendrocrepidine C & Alkaloids & D. crepidatum & $\begin{array}{l}\text { Anti-inflammatory (NO: } \mathrm{IC}_{50} \\
29.7 \mu \mathrm{M} \text { ) }\end{array}$ & [10] \\
\hline 95 & Dendrocrepidine D & Alkaloids & D. crepidatum & $\begin{array}{l}\text { Anti-inflammatory (NO: } \mathrm{IC}_{50} \\
40.1 \mu \mathrm{M})\end{array}$ & {$[10]$} \\
\hline 96 & Dendrocrepidine E & Alkaloids & D. crepidatum & $\begin{array}{l}\text { Anti-inflammatory (NO: } \mathrm{IC}_{50} \\
35.2 \mu \mathrm{M} \text { ) }\end{array}$ & {$[10]$} \\
\hline 97 & (-)-Dendrocrepidine F & Alkaloids & D. crepidatum & $\begin{array}{l}\text { Anti-inflammatory (NO: } \mathrm{IC}_{50} \\
13.3 \mu \mathrm{M} \text { ) }\end{array}$ & [10] \\
\hline 98 & (+)-Dendrocrepidine F & Alkaloids & D. crepidatum & $\begin{array}{l}\text { Anti-inflammatory (NO: } \mathrm{IC}_{50} \\
42.7 \mu \mathrm{M})\end{array}$ & {$[10]$} \\
\hline 99 & Neoechinulin A & Alkaloids & D. loddigesii & $\begin{array}{l}\text { Anti-inflammatory (NO: } \mathrm{IC}_{50} \\
50.0 \mu \mathrm{M})\end{array}$ & [20] \\
\hline 100 & Moupinamide & Alkaloids & D. officinale & Antioxidant (DPPH: $\mathrm{IC}_{50} 53.8 \mu \mathrm{M}$ ) & {$[65]$} \\
\hline 101 & Dihydroferuloyltyramine & Alkaloids & D. officinale & $\begin{array}{l}\text { Antioxidant (DPPH: } \mathrm{IC}_{50} 35.8 \mu \mathrm{g} / \\
\mathrm{mL} \text { ) }\end{array}$ & {$[65]$} \\
\hline 102 & Apigenin & Flavonoids & D. williamsonii & Antioxidant (DPPH: $\mathrm{IC}_{50} 19.3 \mu \mathrm{M}$ ) & {$[74]$} \\
\hline
\end{tabular}


Table 2 (continued)

\begin{tabular}{|c|c|c|c|c|c|}
\hline No. & Name & Type & Source & Pharmacological activities $^{\mathrm{a}}$ & References \\
\hline 103 & Quercetin & Flavonoids & D. tosaense & Antioxidant & [54] \\
\hline 104 & Naringenin & Flavonoids & D. loddigesii & $\begin{array}{l}\text { Anti-inflammatory (NO: } \mathrm{IC}_{50} \\
26.9 \mu \mathrm{M})\end{array}$ & {$[20]$} \\
\hline 105 & $3^{\prime}, 5,5^{\prime}, 7-$ Tetrahydroxyflavanone & Flavonoids & D. officinale & Antioxidant (DPPH: $\mathrm{IC}_{50} 29.8 \mu \mathrm{M}$ ) & {$[65]$} \\
\hline 106 & $\begin{array}{l}\text { 5,4'-Dihydroxy-7,3', } 5^{\prime}- \\
\text { trimethoxyflavanone }\end{array}$ & Flavonoids & D. loddigesii & $\begin{array}{l}\text { Anti-inflammatory (NO: } \mathrm{IC}_{50} \\
24.9 \mu \mathrm{M} \text { ) and antioxidant (DPPH: } \\
\left.\mathrm{IC}_{50} 78.9 \mu \mathrm{g} / \mathrm{mL}\right)\end{array}$ & {$[20]$} \\
\hline 107 & $\begin{array}{l}\text { 5,7,4'-Trihydroxy-3', } 5^{\prime}- \\
\text { dimethoxyflavanone }\end{array}$ & Flavonoids & D. loddigesii & $\begin{array}{l}\text { Anti-inflammatory (NO: } \mathrm{IC}_{50} \\
19.1 \mu \mathrm{M})\end{array}$ & {$[20]$} \\
\hline 108 & Vicenin 2 (vicenin II) & Flavonoids & D. officinale & $\begin{array}{l}\text { Anti-inflammatory (TNF- } \alpha \text { : } \text { IC }_{50} \\
\left.6.8 \mu \mathrm{M} \text {; NO: } \mathrm{IC}_{50} 3.9 \mu \mathrm{M}\right)\end{array}$ & {$[25,26]$} \\
\hline 109 & Dihydroconiferyl alcohol & Phenylpropanoids & D. nobile & Antioxidant (DPPH: $\mathrm{IC}_{50} 50.9 \mu \mathrm{M}$ ) & {$[56]$} \\
\hline 110 & Coniferylaldehyde & Phenylpropanoids & D. nobile & Antioxidant $\left(\mathrm{IC}_{50} 22.8 \mu \mathrm{g} / \mathrm{mL}\right)$ & {$[56]$} \\
\hline \multirow[t]{2}{*}{111} & Ferulic acid & Phenylpropanoids & D. officinale & Antioxidant (DPPH: $\mathrm{IC}_{50} 64.9 \mu \mathrm{M}$ ) & {$[65]$} \\
\hline & & & D. secundum & Antioxidant (DPPH: $\mathrm{IC}_{50} 37.5 \mu \mathrm{M}$ ) & [70] \\
\hline 112 & 6-Feruloyloxyhexanoic ester & Phenylpropanoids & Dendrobium cv. Sonia & $\begin{array}{l}\text { Anti-inflammatory (NO: } \mathrm{IC}_{50} \\
29.6 \mu \mathrm{M})\end{array}$ & [29] \\
\hline 113 & $\begin{array}{l}\text { Threo-7-O-ethyl-9- } O \text {-(4-hydroxy- } \\
\text { phenyl)propionyl-guaiacylglycerol }\end{array}$ & Phenylpropanoids & D. loddigesii & Antioxidant & [49] \\
\hline \multirow[t]{2}{*}{114} & Dihydroconiferyl dihydro- $p$-cou- & Phenylpropanoids & D. loddigesii & Antioxidant & [49] \\
\hline & marate & & D. officinale & Antioxidant (DPPH: $\mathrm{IC}_{50} 78.2 \mu \mathrm{M}$ ) & {$[65]$} \\
\hline 115 & $p$-Hydroxyphenethyl trans-ferulate & Phenylpropanoids & D. loddigesii & Antioxidant & [49] \\
\hline 116 & $n$-Tetracosyl trans-ferulate & Phenylpropanoids & D. moniliforme & Antioxidant & {$[54]$} \\
\hline 117 & $n$-Pentacosyl trans-ferulate & Phenylpropanoids & D. moniliforme & Antioxidant & {$[54]$} \\
\hline 118 & (-)-Pinoresinol & Lignans & D. loddigesii & $\begin{array}{l}\text { Anti-inflammatory (NO: } \mathrm{IC}_{50} \\
89.5 \mu \mathrm{M} \text { ) }\end{array}$ & [19] \\
\hline \multirow[t]{2}{*}{119} & Pinoresinol & Lignans & D. nobile & Antioxidant (DPPH: $\mathrm{IC}_{50} 60.6 \mu \mathrm{M}$ ) & [57] \\
\hline & & & Dendrobium cv. Sonia & $\begin{array}{l}\text { Anti-inflammatory (NO: } \mathrm{IC}_{50} \\
26.3 \mu \mathrm{M})\end{array}$ & [29] \\
\hline 120 & (-)-Medioresinol & Lignans & D. loddigesii & $\begin{array}{l}\text { Anti-inflammatory (NO: } \mathrm{IC}_{50} \\
5.0 \mu \mathrm{M})\end{array}$ & [19] \\
\hline 121 & Medioresinol & Lignans & D. nobile & Antioxidant (DPPH: $\mathrm{IC}_{50} 27.9 \mu \mathrm{M}$ ) & [57] \\
\hline \multirow[t]{4}{*}{122} & Syringaresinol & Lignans & D. loddigesii & $\begin{array}{l}\text { Anti-inflammatory (NO: } \mathrm{IC}_{50} \\
1.9 \mu \mathrm{M}) \text { and antioxidant }\left(\mathrm{IC}_{50}\right. \\
31.1 \mu \mathrm{M})\end{array}$ & {$[20]$} \\
\hline & & & D. nobile & Antioxidant (DPPH: $\mathrm{IC}_{50} 9.8 \mu \mathrm{M}$ ) & [57] \\
\hline & & & D. secundum & Antioxidant (DPPH: $\mathrm{IC}_{50} 11.4 \mu \mathrm{M}$ ) & {$[70]$} \\
\hline & & & Dendrobium cv. Sonia & $\begin{array}{l}\text { Anti-inflammatory (NO: } \mathrm{IC}_{50} \\
27.7 \mu \mathrm{M})\end{array}$ & [29] \\
\hline 123 & Lirioresinol A & Lignans & D. nobile & Antioxidant (DPPH: $\mathrm{IC}_{50} 30.9 \mu \mathrm{M}$ ) & {$[57]$} \\
\hline 124 & Sesqui-illisimonan A & Lignans & Dendrobium cv. Sonia & $\begin{array}{l}\text { Anti-inflammatory (NO: } \mathrm{IC}_{50} \\
31.6 \mu \mathrm{M})\end{array}$ & [29] \\
\hline 125 & Dendrocoumarin & Benzocoumarins & D. nobile & $\begin{array}{l}\text { Antibacterial (Staphylococcus } \\
\text { aureus: MIC } 2.5 \mu \mathrm{g} / \mathrm{mL} ; \text { Micro- } \\
\text { coccus tetragenus: MIC } 5.0 \mu \mathrm{g} / \\
\mathrm{mL} \text { ) }\end{array}$ & {$[36]$} \\
\hline 126 & Itolide A & Benzocoumarins & D. nobile & $\begin{array}{l}\text { Antibacterial (Staphylococcus } \\
\text { aureus: MIC } 2.5 \mu \mathrm{g} / \mathrm{mL} ; \text { Micro- } \\
\text { coccus tetragenus: MIC } 5.0 \mu \mathrm{g} / \\
\mathrm{mL} \text { ) }\end{array}$ & {$[36]$} \\
\hline \multirow[t]{2}{*}{127} & Dendroflorin & Fluorenones & D. nobile & $\begin{array}{l}\text { Anti-inflammatory (NO: } \mathrm{IC}_{50} \\
13.4 \mu \mathrm{g} / \mathrm{mL} \text { ) and antioxidant } \\
\text { (DPPH: } \mathrm{IC}_{50} 16.2 \mu \mathrm{M} \text { ) }\end{array}$ & {$[23]$} \\
\hline & & & D. palpebrae & Antioxidant & [69] \\
\hline
\end{tabular}


Table 2 (continued)

\begin{tabular}{|c|c|c|c|c|c|}
\hline No. & Name & Type & Source & Pharmacological activities $^{\mathrm{a}}$ & References \\
\hline 128 & Nobilone & Fluorenones & D. nobile & $\begin{array}{l}\text { Anti-inflammatory (NO: } \mathrm{IC}_{50} \\
38.1 \mu \mathrm{g} / \mathrm{mL} \text { ) }\end{array}$ & [23] \\
\hline 129 & $\begin{array}{l}\text { 3,6,9-Trihydroxy-3,4-dihydroanthra- } \\
\text { cen-1-(2H)-one }\end{array}$ & Anthracenes & D. loddigesii & $\begin{array}{l}\text { Anti-inflammatory (NO: } \mathrm{IC}_{50} \\
43.8 \mu \mathrm{M} \text { ) and antioxidant (DPPH: } \\
\left.\mathrm{IC}_{50} 22.8 \mu \mathrm{M}\right)\end{array}$ & {$[20]$} \\
\hline 130 & 3-Hydroxy-4-methoxyphenylethanol & Phenylethanoids & D. nobile & Antioxidant (DPPH: $\left.\mathrm{IC}_{50} 64.5 \mu \mathrm{M}\right)$ & {$[56]$} \\
\hline \multirow[t]{2}{*}{131} & Syringic acid & Benzoic acid derivatives & D. nobile & Antioxidant (DPPH: $\mathrm{IC}_{50} 8.1 \mu \mathrm{M}$ ) & {$[56]$} \\
\hline & & & D. officinale & Antioxidant (DPPH: $\mathrm{IC}_{50} 36.5 \mu \mathrm{M}$ ) & {$[65]$} \\
\hline
\end{tabular}

${ }^{\mathrm{a}}$ Pharmacological data with $\mathrm{EC}_{50}, \mathrm{IC}_{50}$, or MIC values are presented herein. Other data can be found in the text

allergies, while antimicrobial activities are associated with treatments of boils, impetigo, and pimples.

Anti-inflammatory and antioxidant activities are the most common activities of Dendrobium extracts and compounds. One hundred thirty-one compounds from Dendrobium plants have been reported to possess anti-inflammatory, antimicrobial, antioxidant, antiaging, anti-psoriasis, and tyrosinaseinhibitory activities (Table 2). These compounds include bibenzyls (1-44, Figs. 1 and 2), phenanthrenes (45-79, Fig. 3), alkaloids (80-101, Fig. 4), flavonoids (102-108), phenylpropanoids (109-117), lignans (118-124), and others (125-131, Fig. 5).

\subsection{Anti-Inflammatory Activity}

Skin inflammation is the most common complaint of those suffering from dermatological diseases. Inflammatory skin diseases are divided into acute and chronic conditions. Acute skin inflammation is associated with occasional rashes, itching and skin redness and may be caused by ultraviolet or ionizing radiation, allergens or chemical irritants. Chronic inflammatory skin diseases include atopic dermatitis (such as eczema), seborrheic dermatitis, psoriasis, and rosacea. Chronic inflammatory skin diseases may lead to significant and serious disruption of skin immunity [8].

\subsubsection{Dendrobium chrysanthum Wall. ex Lindl.}

A phenanthrene, dendrochrysanene (75), was isolated from the stems of $D$. chrysanthum collected from Yunnan, China. This compound significantly suppressed the mRNA levels of TNF- $\alpha$, IL- 8 , IL10, and iNOS in murine peritoneal macrophages at a concentration of $11.2 \mu \mathrm{g} / \mathrm{mL}$. The compound may be a potentially useful new anti-inflammatory agent [9]

\subsubsection{Dendrobium crepidatum Lindl. \& Paxton}

A research was conducted on D. crepidatum stems collected from Yunnan, China. Total alkaloids (yield, 2.3\%) were obtained from $D$. crepidadum stems, which exhibited inhibitory effects on nitric oxide (NO) production in lipopolysaccharide (LPS)-activated mouse peritoneal macrophages, with an $\mathrm{IC}_{50}$ value of $18.7 \mu \mathrm{g} / \mathrm{mL}$. The active alkaloids were found to be dendrocrepidine $\mathrm{A}\left(\mathbf{8 5} ; \mathrm{IC}_{50}, 39.8 \mu \mathrm{M}\right)$, dendrocrepidine $\mathrm{B}\left(\mathbf{9 3} ; \mathrm{IC}_{50}, 51.8 \mu \mathrm{M}\right)$, dendrocrepidine $\mathrm{C}(\mathbf{9 4}$; $\left.\mathrm{IC}_{50}, 29.7 \mu \mathrm{M}\right)$, dendrocrepidine $\mathrm{D}\left(\mathbf{9 5} ; \mathrm{IC}_{50}, 40.1 \mu \mathrm{M}\right)$, dendrocrepidine $\mathrm{E}\left(\mathbf{9 6} ; \mathrm{IC}_{50}, 35.2 \mu \mathrm{M}\right),( \pm)$-dendrocrepidine $\mathrm{F}$ $\left(\mathrm{IC}_{50}, 38.4 \mu \mathrm{M}\right),(-)$-dendrocrepidine $\mathrm{F}\left(\mathbf{9 7} ; \mathrm{IC}_{50}, 13.3 \mu \mathrm{M}\right)$, and (+)-dendrocrepidine $\mathrm{F}\left(\mathbf{9 8} ; \mathrm{IC}_{50}, 42.7 \mu \mathrm{M}\right)$ [10]. In another study, the racemic mixture of $( \pm)$-homocrepidine A was separated into a pair of enantiomers, $(+)$-homocrepidine A (82) and (-)-homocrepidine A (83). ( \pm )-Homocrepidine $\mathrm{A},(+)$-homocrepidine $\mathrm{A},(-)$-homocrepidine $\mathrm{A}$, and homocrepidine B $(\mathbf{8 4})$ also inhibited NO production with $\mathrm{IC}_{50}$ values of $14.7,3.6,22.8$, and $27.6 \mu \mathrm{M}$, respectively, as compared with the positive control indomethacin $\left(\mathrm{IC}_{50}\right.$, $42.2 \mu \mathrm{M})$ [11]. In a very recent study, $( \pm)$-dendrocrepidine A $\left(\mathrm{IC}_{50}, 63.8 \mu \mathrm{M}\right),(+)$-dendrocrepidine $\mathrm{A}\left(\mathbf{8 5} ; \mathrm{IC}_{50}, 16.1 \mu \mathrm{M}\right)$, dendrocrepidine $\mathrm{B}\left(\mathbf{8 6} ; \mathrm{IC}_{50}, 14.3 \mu \mathrm{M}\right),( \pm)$-crepidine $\left(\mathrm{IC}_{50}\right.$, $51.1 \mu \mathrm{M}),(-)$-crepidine $\left(87 ; \mathrm{IC}_{50}, 29.9 \mu \mathrm{M}\right),(+)$-crepidine $\left(\mathbf{8 8} ; \mathrm{IC}_{50}, 81.9 \mu \mathrm{M}\right),( \pm)$-dendrocrepidine $\mathrm{A}\left(\mathrm{IC}_{50}, 27.5 \mu \mathrm{M}\right)$, (-)-dendrocrepidine $\mathrm{A}\left(\mathbf{8 9} ; \mathrm{IC}_{50}, 18.5 \mu \mathrm{M}\right),(+)$-dendrocrepidine $\mathrm{A}\left(\mathbf{9 0} ; \mathrm{IC}_{50}, 30.2 \mu \mathrm{M}\right),( \pm)$-isocrepidamine $\left(\mathrm{IC}_{50}\right.$, $27.4 \mu \mathrm{M}),(-)$-isocrepidamine $\left(91 ; \mathrm{IC}_{50}, 16.3 \mu \mathrm{M}\right)$, and (+)-isocrepidamine $\left(\mathbf{9 2} ; \mathrm{IC}_{50}, 73.0 \mu \mathrm{M}\right)$ also exhibited inhibitory effects on $\mathrm{NO}$ production. Dexamethasone $\left(\mathrm{IC}_{50}\right.$, $47.0 \mu \mathrm{M}$ ) was used as a positive control [12].

\subsubsection{Dendrobium denneanum Kerr.}

A series of phenanthrene derivatives were isolated from $D$. denneanum stems collected from Sichuan, China, and 2,5-dihydroxy-4-methoxy-phenanthrene $2-O-\beta$-D-glucopyranoside $\left(\mathbf{7 6} ; \mathrm{IC}_{50}, 4.6 \mu \mathrm{M}\right)$, 2,5-dihydroxy-4-methoxy-phenanthrene $2-O-\beta$-Dapiofuranosyl- $(1 \rightarrow 6)$ - $\beta$-D-glucopyranoside $\left(77 ; \mathrm{IC}_{50}\right.$, $16.9 \mu \mathrm{M}), 2,5$-dihydroxy-4-methoxy-phenanthrene 2 - $O$ - $\alpha$-L-rhamnopyranosyl- $(1 \rightarrow 6)$ - $\beta$-D-glucopyranoside (78; $\left.\mathrm{IC}_{50}, 41.5 \mu \mathrm{M}\right),(S)$-5-methoxy-2,4,7,9-tetrahydroxy-9,10-dihydrophenanthrene $\left(66 ; \mathrm{IC}_{50}, 3.1 \mu \mathrm{M}\right)$, 
<smiles>[R5]c1ccc(CCc2cc([R])c([R])c([R])c2)cc1[R4]</smiles>
1: $\mathrm{R}_{1}=\mathrm{R}_{4}=\mathrm{OH}, \mathrm{R}_{2}=\mathrm{R}_{5}=\mathrm{H}, \mathrm{R}_{3}=\mathrm{OMe}$
2: $\mathrm{R}_{1}=\mathrm{R}_{3}=\mathrm{R}_{4}=\mathrm{OH}, \mathrm{R}_{2}=\mathrm{R}_{5}=\mathrm{H}$
3: $\mathrm{R}_{1}=\mathrm{R}_{5}=\mathrm{OH}, \mathrm{R}_{2}=\mathrm{R}_{4}=\mathrm{H}, \mathrm{R}_{3}=\mathrm{OMe}$
4: $\mathrm{R}_{1}=\mathrm{OH}, \mathrm{R}_{2}=\mathrm{R}_{4}=\mathrm{H}, \mathrm{R}_{3}=\mathrm{R}_{5}=\mathrm{OMe}$
5: $\mathrm{R}_{1}=\mathrm{R}_{3}=\mathrm{OMe}, \mathrm{R}_{2}=\mathrm{R}_{5}=\mathrm{OH}, \mathrm{R}_{4}=\mathrm{H}$
6: $\mathrm{R}_{1}=\mathrm{R}_{4}=\mathrm{OH}, \mathrm{R}_{2}=\mathrm{R}_{3}=\mathrm{OMe}, \mathrm{R}_{5}=\mathrm{H}$
7: $\mathrm{R}_{1}=\mathrm{R}_{5}=\mathrm{OH}, \mathrm{R}_{2}=\mathrm{H}, \mathrm{R}_{3}=\mathrm{R}_{4}=\mathrm{OMe}$
8: $\mathrm{R}_{1}=\mathrm{R}_{3}=\mathrm{R}_{5}=\mathrm{OH}, \mathrm{R}_{2}=\mathrm{H}, \mathrm{R}_{4}=\mathrm{OMe}$

9: $\mathrm{R}_{1}=\mathrm{R}_{3}=\mathrm{R}_{4}=\mathrm{OMe}, \mathrm{R}_{2}=\mathrm{R}_{5}=\mathrm{OH}$

10: $\mathrm{R}_{1}=\mathrm{R}_{2}=\mathrm{OH}, \mathrm{R}_{3}=\mathrm{R}_{4}=\mathrm{OMe}, \mathrm{R}_{5}=\mathrm{H}$

11: $\mathrm{R}_{1}=\mathrm{R}_{3}=\mathrm{R}_{4}=\mathrm{R}_{5}=\mathrm{OMe}, \mathrm{R}_{2}=\mathrm{OH}$

12: $R_{1}=R_{2}=R_{4}=R_{5}=O H, R_{3}=O M e$

13: $\mathrm{R}_{1}=\mathrm{R}_{2}=\mathrm{R}_{5}=\mathrm{OH}, \mathrm{R}_{3}=\mathrm{R}_{4}=\mathrm{OMe}$

14: $\mathrm{R}_{1}=\mathrm{R}_{2}=\mathrm{R}_{3}=\mathrm{R}_{5}=\mathrm{OMe}, \mathrm{R}_{4}=\mathrm{OH}$

15: $R_{1}=R_{2}=R_{3}=R_{4}=O M e, R_{5}=O H$

16: $R_{1}=R_{2}=R_{3}=R_{4}=R_{5}=O M e$<smiles>COC1=CC(O)(CCc2ccc(O)c(OC)c2)C=C(OC)C1=O</smiles><smiles>[R]Oc1ccc(C[C@@H](O[R])c2cc(O)c(O)c(OC)c2)cc1</smiles>

18: $R=H, R^{\prime}=M e$

19: $\mathrm{R}=\mathrm{H}, \mathrm{R}^{\prime}=\mathrm{Et}$

20: $R=M e, R^{\prime}=H$

17<smiles>[R]c1ccc(CC([R3])c2cc([R])c(O)c(OC)c2)cc1OC</smiles>

21: $\mathrm{R}_{1}=\mathrm{OMe}, \mathrm{R}_{2}=\mathrm{R}_{3}=\mathrm{OH}$

22: $\mathrm{R}_{1}=\mathrm{OH}, \mathrm{R}_{2}=\mathrm{H}, \mathrm{R}_{3}=\mathrm{OMe}$

23: $\mathrm{R}_{1}=\mathrm{R}_{3}=\mathrm{OMe}, \mathrm{R}_{2}=\mathrm{OH}$

24: $\mathrm{R}_{1}=\mathrm{R}_{2}=\mathrm{R}_{3}=\mathrm{OMe}$<smiles>COc1cc(C[C@@H](OC)c2cc(OC)c(O)c(OC)c2)ccc1O</smiles><smiles>COc1cc(C(=O)C(=O)c2cc(O)c(O)c(OC)c2)ccc1O</smiles><smiles>COc1cc(CCc2ccc(O)c(OC)c2)c2c(c1)OC(c1ccc(O)c(OC)c1)C(O)C2</smiles><smiles>COc1ccc([C@H]2Oc3cc(O)cc(CCc4ccc(O)c(OC)c4)c3C[C@@H]2O)cc1O</smiles>

Fig. 1 Chemical structures of pharmacologically active bibenzyls (1-28) from Dendrobium plants (I)

(S)-4-methoxy-2,5,7,9-tetrahydroxy-9,10-dihydrophenanthrene $\left(67 ; \mathrm{IC}_{50}, 4.2 \mu \mathrm{M}\right),(9 R)-1,2,5,9$-tetrahydroxy9,10-dihydrophenanthrene 5- $O$ - $\beta$-D-glucopyranoside (79; $\mathrm{IC}_{50}, 0.7 \mu \mathrm{M}$ ), 4-methoxyphenanthrene-2,5-diol (moscatin, 45; $\left.\mathrm{IC}_{50}, 6.3 \mu \mathrm{M}\right),(S)$-4-methoxy-9,10-dihydrophenanthrene-2,5,9-triol $\left(65 ; \mathrm{IC}_{50}, 27.4 \mu \mathrm{M}\right)$, 2-methoxy9,10-dihydrophenanthrene-4,5-diol (57; $\left.\mathrm{IC}_{50}, 7.6 \mu \mathrm{M}\right)$, and 9,10-dihydrophenanthrene-2,4,7-triol $\left(\mathbf{5 6} ; \mathrm{IC}_{50}, 32.7 \mu \mathrm{M}\right)$ inhibited NO production in LPS-activated mouse macrophage RAW264.7 cells, as compared with the positive control curcumin $\left(\mathrm{IC}_{50}, 6.2 \mu \mathrm{M}\right)$ [13].

\subsubsection{Dendrobium findlayanum C.S.P. Parish \& Rchb.f.}

Seco-dendrobine-type alkaloids and phenolics were isolated from $D$. findlayanum stems collected from Yunnan, China. Bibenzyl 6"-de- $O$-methyldendrofindlaphenol A (38) inhibited NO production in RAW 264.7 cells with an $\mathrm{IC}_{50}$ value of $21.4 \mu \mathrm{M}$. MG-132 ( $\left.\mathrm{IC}_{50}, 0.2 \mu \mathrm{M}\right)$ was used as a positive control [14]. 
<smiles>[R]c1ccc(CCc2cc(OC)c(O)c3c2C(Cc2ccc([R2])c([R])c2)c2cc(O)c(O)c(OC)c2O3)cc1[R]</smiles><smiles></smiles><smiles>COc1cc(CC2c3cc(O)c(O)c(OC)c3Oc3cc(O)c4c(c32)CCc2cc(O)ccc2-4)ccc1O</smiles>

29: $\mathrm{R}_{1}=\mathrm{R}_{3}=\mathrm{H}, \mathrm{R}_{2}=\mathrm{R}_{4}=\mathrm{OMe}$

30: $\mathrm{R}_{1}=\mathrm{R}_{3}=\mathrm{H}, \mathrm{R}_{2}=\mathrm{OH}, \mathrm{R}_{4}=\mathrm{OMe}$

31: $\mathrm{R}_{1}=\mathrm{R}_{3}=\mathrm{H}, \mathrm{R}_{2}=\mathrm{OMe}, \mathrm{R}_{4}=\mathrm{OH}$

32: $\mathrm{R}_{1}=\mathrm{R}_{3}=\mathrm{H}, \mathrm{R}_{2}=\mathrm{R}_{4}=\mathrm{OH}$

33: $\mathrm{R}_{1}=\mathrm{R}_{3}=\mathrm{OMe}, \mathrm{R}_{2}=\mathrm{R}_{4}=\mathrm{H}$<smiles>COc1cc(C[C@H](O)[C@H](CO)Oc2c(OC)cc(CCc3ccc(O)cc3)cc2OC)ccc1O</smiles><smiles>COc1ccc(CCc2cc(OC)c3c(c2)O[C@H](c2cc(O)c(O)c(OC)c2)[C@H](c2ccc(OC)cc2)O3)cc1</smiles>

39<smiles>COc1cc([C@H]2Oc3cc(CCc4ccc(O)cc4)cc(OC)c3O[C@@H]2c2ccc(O)cc2)cc(O)c1O</smiles><smiles>COc1cc([C@@H]2Oc3cc(CCc4ccc(O)cc4)cc(OC)c3O[C@H]2c2ccc(O)cc2)cc(O)c1O</smiles>

40<smiles>[R]c1cc([C@H]2Oc3cc(CCc4ccc(O)cc4)cc(OC)c3O[C@@H]2CO)cc(OC)c1O</smiles>

Fig. 2 Chemical structures of pharmacologically active bibenzyls (29-44) from Dendrobium plants (II)

\subsubsection{Dendrobium heterocarpum Lindl.}

3-Hydroxy-4',5-dimethoxybibenzyl (4), gigantol (7), and dendrocandin I (39) were isolated from whole plants of D. heterocarpum collected from Yunnan, China, and these compounds reduced nitric oxide (NO) production in LPSactivated mouse macrophage RAW264.7 cells. At a concentration of $25 \mu \mathrm{M}$, the inhibition percentages of these three compounds were 51,52 , and $59 \%$, respectively. NGMonomethyl-L-arginine (L-NMMA) was used as a positive control $\left(\mathrm{IC}_{50}, 34 \mu \mathrm{M}\right)[15]$.

\subsubsection{Dendrobium huoshanense Z.Z. Tang \& S.J. Cheng}

Dendrobium huoshanense Z.Z. Tang \& S.J. Cheng was not accepted by "Plants of the World online." However, because 
<smiles>[R]Cc1cc2ccc3c([R])c([R6])c([R])c([R])c3c2c([R])c1[R]</smiles>

45: $\mathrm{R}_{1}=\mathrm{R}_{2}=\mathrm{R}_{5}=\mathrm{R}_{7}=\mathrm{H}, \mathrm{R}_{3}=\mathrm{R}_{6}=\mathrm{OH}, \mathrm{R}_{4}=\mathrm{OMe}$

46: $\mathrm{R}_{1}=\mathrm{R}_{3}=\mathrm{OMe}, \mathrm{R}_{2}=\mathrm{R}_{5}=\mathrm{R}_{6}=\mathrm{R}_{7}=\mathrm{H}, \mathrm{R}_{4}=\mathrm{OH}$

47: $\mathrm{R}_{1}=\mathrm{R}_{6}=\mathrm{OH}, \mathrm{R}_{2}=\mathrm{R}_{3}=\mathrm{R}_{7}=\mathrm{OMe}, \mathrm{R}_{4}=\mathrm{R}_{5}=\mathrm{H}$

48: $\mathrm{R}_{1}=\mathrm{R}_{2}=\mathrm{R}_{4}=\mathrm{OH}, \mathrm{R}_{3}=\mathrm{OMe}, \mathrm{R}_{5}=\mathrm{R}_{6}=\mathrm{R}_{7}=\mathrm{H}$

49: $\mathrm{R}_{1}=\mathrm{R}_{6}=\mathrm{OH}, \mathrm{R}_{2}=\mathrm{R}_{4}=\mathrm{R}_{5}=\mathrm{R}_{7}=\mathrm{H}, \mathrm{R}_{3}=\mathrm{OMe}$

50: $\mathrm{R}_{1}=\mathrm{R}_{3}=\mathrm{OMe}, \mathrm{R}_{2}=\mathrm{R}_{6}=\mathrm{OH}, \mathrm{R}_{4}=\mathrm{R}_{5}=\mathrm{R}_{7}=\mathrm{H}$

51: $\mathrm{R}_{1}=\mathrm{R}_{4}=\mathrm{OH}, \mathrm{R}_{2}=\mathrm{R}_{3}=\mathrm{R}_{7}=\mathrm{OMe}, \mathrm{R}_{5}=\mathrm{R}_{6}=\mathrm{H}$<smiles>[R]c1cc2c(c([R])c1[R])-c1c(cc([R6])c([R])c1[R])CC2</smiles>

54: $\mathrm{R}_{1}=\mathrm{R}_{4}=\mathrm{OH}, \mathrm{R}_{2}=\mathrm{R}_{3}=\mathrm{R}_{5}=\mathrm{H}, \mathrm{R}_{6}=\mathrm{OMe}$

55: $\mathrm{R}_{1}=\mathrm{R}_{4}=\mathrm{OH}, \mathrm{R}_{2}=\mathrm{R}_{5}=\mathrm{R}_{6}=\mathrm{H}, \mathrm{R}_{3}=\mathrm{OMe}$

56: $\mathrm{R}_{1}=\mathrm{R}_{4}=\mathrm{R}_{6}=\mathrm{OH}, \mathrm{R}_{2}=\mathrm{R}_{3}=\mathrm{R}_{5}=\mathrm{H}$

57: $\mathrm{R}_{1}=\mathrm{R}_{2}=\mathrm{R}_{5}=\mathrm{H}, \mathrm{R}_{3}=\mathrm{R}_{4}=\mathrm{OH}, \mathrm{R}_{6}=\mathrm{OMe}$

58: $\mathrm{R}_{1}=\mathrm{R}_{6}=\mathrm{OH}, \mathrm{R}_{2}=\mathrm{R}_{4}=\mathrm{R}_{5}=\mathrm{H}, \mathrm{R}_{3}=\mathrm{OMe}$

59: $\mathrm{R}_{1}=\mathrm{R}_{4}=\mathrm{R}_{6}=\mathrm{OH}, \mathrm{R}_{2}=\mathrm{R}_{5}=\mathrm{H}, \mathrm{R}_{3}=\mathrm{OMe}$

60: $\mathrm{R}_{1}=\mathrm{R}_{6}=\mathrm{OH}, \mathrm{R}_{2}=\mathrm{R}_{3}=\mathrm{OMe}, \mathrm{R}_{4}=\mathrm{R}_{5}=\mathrm{H}$

61: $\mathrm{R}_{1}=\mathrm{R}_{3}=\mathrm{OMe}, \mathrm{R}_{2}=\mathrm{R}_{6}=\mathrm{OH}, \mathrm{R}_{4}=\mathrm{R}_{5}=\mathrm{H}$

62: $\mathrm{R}_{1}=\mathrm{R}_{2}=\mathrm{R}_{4}=\mathrm{OH}, \mathrm{R}_{3}=\mathrm{OMe}, \mathrm{R}_{5}=\mathrm{R}_{6}=\mathrm{H}$

63: $\mathrm{R}_{1}=\mathrm{R}_{2}=\mathrm{OMe}, \mathrm{R}_{3}=\mathrm{R}_{6}=\mathrm{OH}, \mathrm{R}_{4}=\mathrm{R}_{5}=\mathrm{H}$<smiles>COc1cc(O)cc2c1-c1c(O)cccc1C(O)C2</smiles><smiles>COC1=CC(=O)c2ccc3cc(OC)cc(O)c3c2C1=O</smiles>

68<smiles>COc1cc(O)cc2c1C1=C(CC2)C(=O)C=CC1=O</smiles><smiles>COc1c(O)cc2c(=O)oc3cc(O)cc4ccc1c2c43</smiles><smiles>COc1cc([C@H]2Oc3cc(OC)c4c(ccc5cccc(O)c54)c3C[C@H]2O)ccc1O</smiles><smiles>COc1cc([C@H]2c3c(cc(O)c4c3ccc3cc(O)ccc34)O[C@@H]2c2cc(OC)c(O)c(OC)c2)ccc1O</smiles><smiles>COc1cc([C@H]2c3c(cc(O)c4c3CCc3cc(O)ccc3-4)O[C@H]2c2cc(O)c(O)c(OC)c2)ccc1O</smiles>

$\mathrm{HO}$

65: $\mathrm{R}_{1}=\mathrm{H}, \mathrm{R}_{2}=\mathrm{OH}, \mathrm{R}_{3}=\mathrm{OMe}$

66: $\mathrm{R}_{1}=\mathrm{R}_{3}=\mathrm{OH}, \mathrm{R}_{2}=\mathrm{OMe}$

67: $\mathrm{R}_{1}=\mathrm{R}_{2}=\mathrm{OH}, \mathrm{R}_{3}=\mathrm{OMe}$<smiles>COc1cc2cc(O)ccc2c2c1C1(CC2=O)C(=O)Oc2cc(O)c3c(c(OC)cc4cc(O)ccc43)c21</smiles><smiles>[R]OC[C@H]1O[C@H](Oc2cc(C)c(-c3c(C)cccc3O)c(OC)c2)[C@H](O)[C@@H](O)[C@@H]1O</smiles>

76: $\mathrm{R}=\mathrm{H}$<smiles>COc1cc(O)cc2c1-c1c(O)cc(O)cc1C[C@H]2O</smiles>

77: $R=\beta-D$-apiofuranosyl

78: $R=\alpha_{-L-\text { rhamnopyranosyl }}$

Fig. 3 Chemical structures of pharmacologically active phenanthrenes (45-79) from Dendrobium plants 
<smiles>CN1CCCC12OC(=O)c1ccccc12</smiles><smiles></smiles>

81

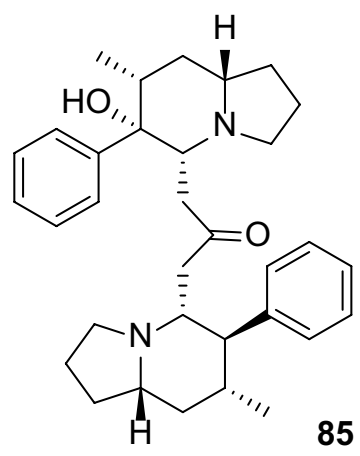

85

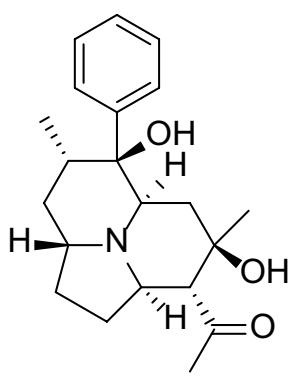

89
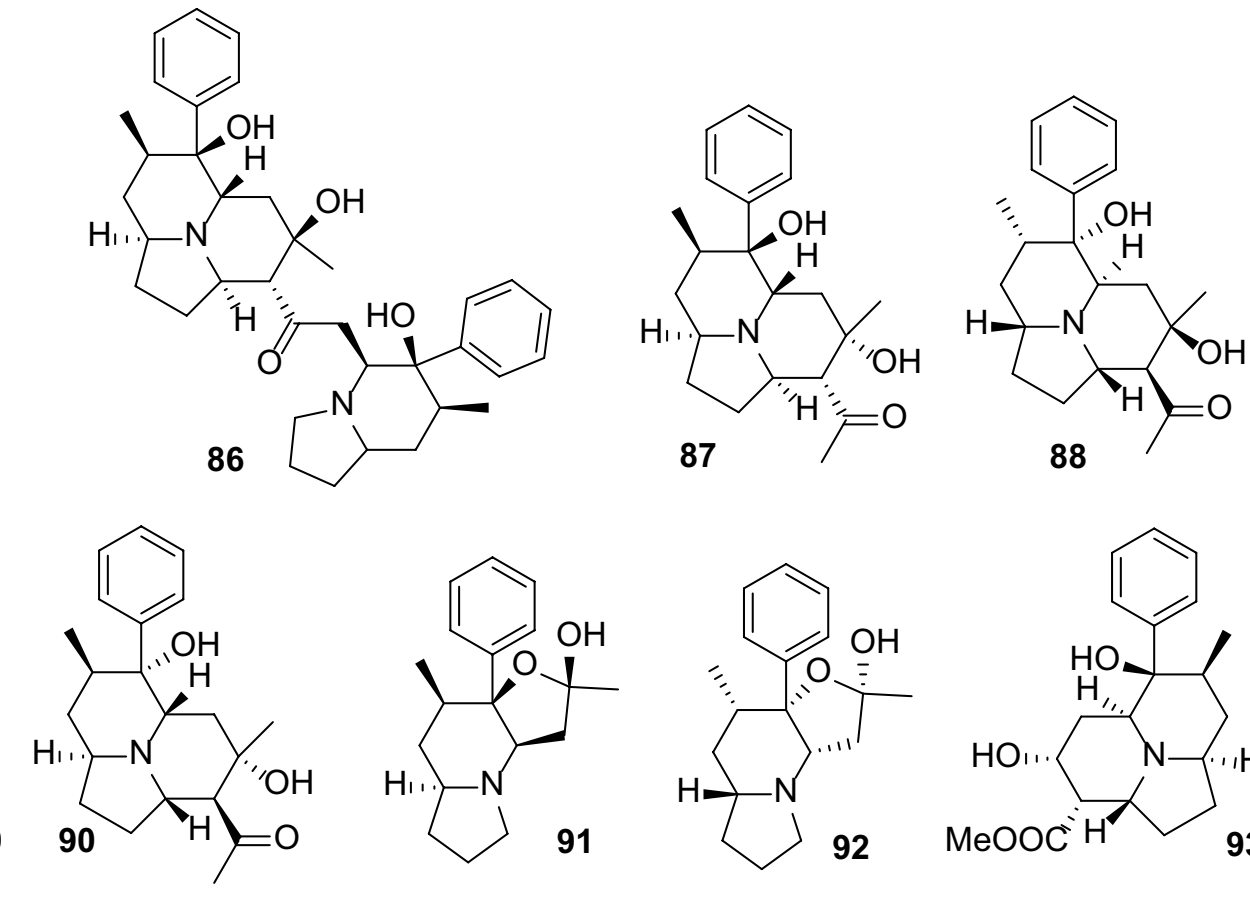<smiles>C[C@@H]1C[C@H]2CCCN2[C@@H]2CC(C)(O)O[C@]12c1ccccc1</smiles>

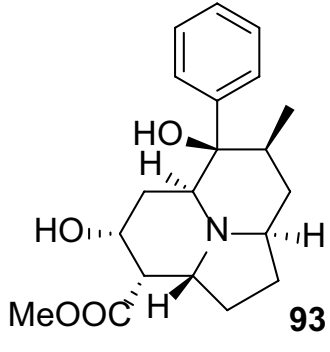<smiles>C[C@H]1C[C@@H](C)[C@@H]2CC[C@H]3C[C@@H](O)C[C@H](N32)[C@@]1(O)c1ccccc1</smiles><smiles>CC1C[C@@H]2CCCN2[C@@H]2CC(=O)O[C@]12c1ccccc1</smiles><smiles>C=CC(C)(C)c1[nH]c2ccccc2c1/C=C1\NC(=O)[C@H](C)NC1=O</smiles><smiles>COc1cc(/C=C/C=O)ccc1O</smiles><smiles>C[C@H](CC1CCCN1)C1(c2ccccc2)CC(CC(=O)C[C@H]2N3CCC[C@H]3C[C@@H](C)C2(O)c2ccccc2)OO1</smiles>
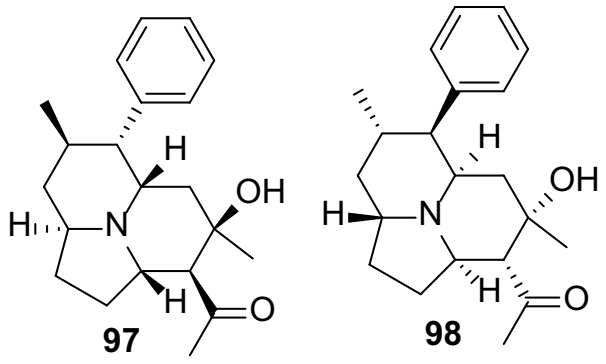

Fig. 4 Chemical structures of pharmacologically active alkaloids (80-101) from Dendrobium plants 
<smiles>[R]c1cc(-c2oc3c([R])c(O)c([R])c(O)c3c(=O)c2[R])ccc1O</smiles>

102: $\mathrm{R}=\mathrm{R}^{\prime}=\mathrm{H}$

103: $\mathrm{R}=\mathrm{OH}, \mathrm{R}^{\prime}=\mathrm{H}$

108: $\mathrm{R}=\mathrm{H}, \mathrm{R}^{\prime}=\mathrm{GlC}$<smiles>[R][R][O-]</smiles>

114<smiles>[R]c1cc(O)c2c(c1)OC(c1cc([R])c([R])c([R])c1)CC2=O</smiles>

104: $\mathrm{R}_{1}=\mathrm{R}_{3}=\mathrm{OH}, \mathrm{R}_{2}=\mathrm{R}_{4}=\mathrm{H}$

105: $\mathrm{R}_{1}=\mathrm{R}_{2}=\mathrm{R}_{4}=\mathrm{OH}, \mathrm{R}_{3}=\mathrm{H}$

106: $R_{1}=R_{2}=R_{4}=O M e, R_{3}=O H$

107: $\mathrm{R}_{1}=\mathrm{R}_{3}=\mathrm{OH}, \mathrm{R}_{2}=\mathrm{R}_{4}=\mathrm{OMe}$<smiles>COc1cc(CCCO)ccc1O</smiles><smiles>[R]C=Cc1ccc(O)c(OC)c1</smiles>

111: $\mathrm{R}=\mathrm{COOH}$<smiles>COc1cc(/C=C/C(=O)OCC(C)(C)C)ccc1O</smiles>

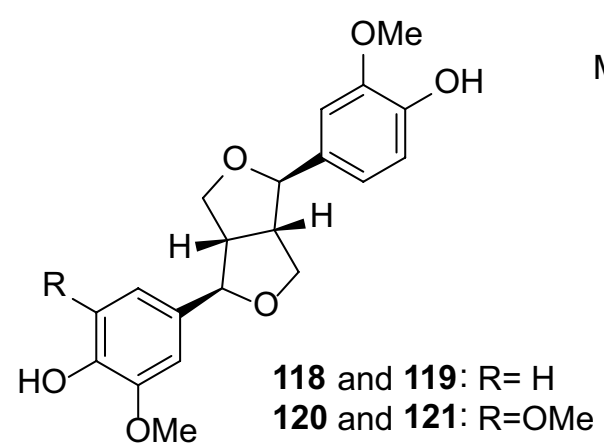<smiles>COc1cc([C@@H]2OC[C@@H]3[C@H]2CO[C@H]3c2cc(OC)c(O)c(OC)c2)cc(OC)c1O</smiles>

113<smiles>CCOC(c1ccc(O)c(OC)c1)[C@H](O)COC(C)=O</smiles>

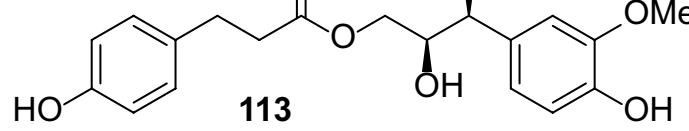<smiles>COc1cc(/C=C/C(=O)OCCc2ccc(O)cc2)ccc1O</smiles>
115<smiles>COc1cc([C@@H]2OC[C@@H]3[C@H]2CO[C@H]3c2cc(OC)c(O)c(OC)c2)cc(OC)c1O</smiles><smiles>[R]c1cc([R])c2c(=O)oc3c(O)cccc3c2c1O</smiles><smiles>C1CCOCC1</smiles><smiles>[R]c1cc([R])c2c(c1[R])C(=O)c1cc(I)cc(OC)c1-2</smiles>

125: $\mathrm{R}=\mathrm{OH}, \mathrm{R}^{\prime}=\mathrm{H}$

126: $\mathrm{R}=\mathrm{H}, \mathrm{R}^{\prime}=\mathrm{OH}$

127: $\mathrm{R}=\mathrm{OH}, \mathrm{R}^{\prime}=\mathrm{H}$

128: $\mathrm{R}=\mathrm{H}, \mathrm{R}^{\prime}=\mathrm{OH}$<smiles></smiles><smiles>COc1ccc(CCO)cc1O</smiles>

Fig. 5 Chemical structures of pharmacologically active flavonoids (102-108), phenylpropanoids (109-117), lignans (118-124) and other active compounds (125-131) from Dendrobium plants 
this name is used in the Chinese Pharmacopoeia (2020 edition) [16], it is cited in this review.

A pilot study was conducted to evaluate the clinical and immunomodulatory effects of an orally administered extract of $D$. huoshanense leaves and stems in children with moderate to severe recalcitrant atopic dermatitis (AD). AD is a common inflammatory skin disorder for which few safe and effective systemic treatments are available. Twenty-seven patients aged 4-18 years with AD who did not respond to topical therapy were treated with polysaccharides derived from $D$. huoshanense for 4 weeks and were followed-up for another 4 weeks. The results showed that the polysaccharide from $D$. huoshanense reduced the levels of some cytokines associated with $\mathrm{AD}$ and had beneficial effects on symptoms. No serious adverse effects occurred when the polysaccharide was administered orally for 4 weeks [17].

\subsubsection{Dendrobium loddigesii Rolfe}

Shihunine (80) was isolated from D. loddigesii stems collected from Yunnan, China. The alkaloid showed antiinflammatory activity using the method of NO production the polysaccharide in RAW 264.7 cells activated by LPS with an $\mathrm{IC}_{50}$ value of $11.5 \mu \mathrm{g} / \mathrm{mL}$. L-NG-monomethyl arginine citrate $\left(\mathrm{IC}_{50} 7.2 \mu \mathrm{g} / \mathrm{mL}\right.$ ) was used as a positive control [18].

Phenanthrenes and bibenzyls were isolated from D. loddigesii stems collected from Guangdong, China. These compounds were evaluated for their inhibitory activities against NO production. Loddigesiinol A $\left(\mathbf{5 2} ; \mathrm{IC}_{50}\right.$, $2.6 \mu \mathrm{M}$ ), moscatin (plicatol B, 45; $\mathrm{IC}_{50}, 6.4 \mu \mathrm{M}$ ), 5-hydroxy2,4-dimethoxyphenanthrene $\left(\mathbf{4 6} ; \mathrm{IC}_{50}, 5.3 \mu \mathrm{M}\right)$, lusianthri$\operatorname{din}\left(54 ; \mathrm{IC}_{50}, 4.6 \mu \mathrm{M}\right)$, rotundatin $\left(64\right.$, plicatol $\mathrm{C}$; $\mathrm{IC}_{50}$, $29.1 \mu \mathrm{M})$, hircinol $\left(\mathbf{5 5} ; \mathrm{IC}_{50}, 29.2 \mu \mathrm{M}\right)$, loddigesiinol B (71; $\left.\mathrm{IC}_{50}, 10.9 \mu \mathrm{M}\right)$, loddigesiinol D $\left(\mathbf{2 6} ; \mathrm{IC}_{50}, 69.7 \mu \mathrm{M}\right)$, (-)-pinoresinol (118; $\left.\mathrm{IC}_{50}, 89.5 \mu \mathrm{M}\right)$, and (-)-medioresinol $\left(120 ; \mathrm{IC}_{50}, 5.0 \mu \mathrm{M}\right)$ inhibited $\mathrm{NO}$ production, as compared with the positive controls aminoguanidine $\left(\mathrm{IC}_{50}, 17.5 \mu \mathrm{M}\right)$ and resveratrol $\left(\mathrm{IC}_{50}, 22.0 \mu \mathrm{M}\right)$ [19].

Fifteen compounds were isolated from a preparation of D. loddigesii originating in Yunnan, China. Chrysotoxol A (74; $\left.\mathrm{IC}_{50}, 10.9 \mu \mathrm{M}\right)$, neoechinulin $\mathrm{A}\left(99 ; \mathrm{IC}_{50}, 50.0 \mu \mathrm{M}\right)$, 3,6,9-trihydroxy-3,4-dihydroanthracen-1-(2H)-one (129; IC $\left._{50}, 43.8 \mu \mathrm{M}\right), 4,4^{\prime}$-dihydroxy-3,5-dimethoxybibenzyl $\left(\mathbf{5} ; \mathrm{IC}_{50}, 49.3 \mu \mathrm{M}\right)$, naringenin $\left(\mathbf{1 0 4} ; \mathrm{IC}_{50}, 26.9 \mu \mathrm{M}\right)$, 5,4'-dihydroxy-7,3',5'-trimethoxyflavanone (106; $\mathrm{IC}_{50}$, $24.9 \mu \mathrm{M}), 5,7,4^{\prime}$-trihydroxy-3',5'-dimethoxyflavanone $\left(\mathrm{IC}_{50}, 19.1 \mu \mathrm{M}\right)$, batatasin III $\left(\mathbf{1} ; \mathrm{IC}_{50}, 21.9 \mu \mathrm{M}\right), 3,3^{\prime}, 5-$ trihydroxybibenzyl $\left(2 ; \mathrm{IC}_{50}, 13.1 \mu \mathrm{M}\right)$, trigonopol B (28; $\left.\mathrm{IC}_{50}, 26.3 \mu \mathrm{M}\right)$, syringaresinol $\left(\mathbf{1 2 2} ; \mathrm{IC}_{50}, 1.9 \mu \mathrm{M}\right)$, 9,10-dihydrophenanthrene-2,4,7-triol $\left(\mathbf{5 6} ; \mathrm{IC}_{50}, 8.6 \mu \mathrm{M}\right)$, loddigesiinol $\mathrm{B}\left(\mathbf{7 1} ; \mathrm{IC}_{50}, 9.9 \mu \mathrm{M}\right)$, loddigesiinol I (72; $\left.\mathrm{IC}_{50}, 7.5 \mu \mathrm{M}\right)$, and loddigesiinol $\mathrm{J}\left(73 ; \mathrm{IC}_{50}, 14.6 \mu \mathrm{M}\right)$ inhibited NO production in RAW 264.7 cells activated by LPS when compared with the positive control L-NMMA $\left(\mathrm{IC}_{50}, 29.0 \mu \mathrm{M}\right)[20]$.

\subsubsection{Dendrobium moniliforme (L.) Sw.}

Denbinobin (68) was isolated from D. moniliforme stems. At $1 \mu \mathrm{M}$, this compound significantly inhibited the formation of TNF- $\alpha$ and prostaglandin $\mathrm{E}_{2}\left(\mathrm{PGE}_{2}\right)$ (about 62 and $43 \%$ inhibition, respectively) in RAW264.7 cells stimulated with $1 \mu \mathrm{g} / \mathrm{mL}$ of LPS. In N9 cells (murine microglial cell line) stimulated with $10 \mathrm{ng} / \mathrm{mL}$ of LPS plus $10 \mathrm{unit} / \mathrm{mL}$ of interferon- $\gamma($ IFN- $\gamma)$, denbinobin $(3 \mu \mathrm{M})$ reduced TNF- $\alpha$ and nitrite formation (about 70 and $44 \%$ inhibition, respectively) [21].

\subsubsection{Dendrobium nobile Lindl.}

An alkaloid, anosmine (81), was isolated from $D$. nobile, which was purchased from Guangzhou, China. The compound exhibited anti-inflammatory activity using the method of NO production inhibition in RAW 264.7 cells activated by LPS with an $\mathrm{IC}_{50}$ value of $16.1 \mu \mathrm{g} / \mathrm{mL}$, which was compared with L-NG-monomethyl arginine citrate $\left(\mathrm{IC}_{50}, 7.2 \mu \mathrm{g} / \mathrm{mL}\right)$ as a positive control [18].

Phenanthrenes from the methanolic extract of $D$. nobile stems were evaluated for their potential to inhibit LPSinduced production of $\mathrm{NO}$ in murine macrophage RAW 264.7 cells. 3,4,8-Trimethoxyphenanthrene-2,5-diol (51; $\left.\mathrm{IC}_{50}, 20.4 \mu \mathrm{M}\right)$, hircinol $\left(55 ; \mathrm{IC}_{50}, 26.4 \mu \mathrm{M}\right)$, erianthridin (60; $\left.\mathrm{IC}_{50}, 19.5 \mu \mathrm{M}\right)$, ephemeranthol A $\left(63 ; \mathrm{IC}_{50}, 12.0 \mu \mathrm{M}\right)$, 5,7-dimethoxyphenanthrene-2,6-diol (50; $\left.\mathrm{IC}_{50}, 35.7 \mu \mathrm{M}\right)$, moscatilin $\left(\mathbf{9} ; \mathrm{IC}_{50}, 27.6 \mu \mathrm{M}\right)$, coelonin $\left(\mathbf{5 8} ; \mathrm{IC}_{50}, 10.2 \mu \mathrm{M}\right)$, flavanthridin $\left(61 ; \mathrm{IC}_{50}, 34.1 \mu \mathrm{M}\right)$, epheneranthol C (62; $\mathrm{IC}_{50}$, $17.6 \mu \mathrm{M})$, lusianthridin $\left(\mathbf{5 4} ; \mathrm{IC}_{50}, 9.6 \mu \mathrm{M}\right)$, and fimbriol $\mathrm{B}$ $\left(\mathbf{4 8} ; \mathrm{IC}_{50}, 28.9 \mu \mathrm{M}\right)$ inhibited $\mathrm{NO}$ production. Aminoguanidine $\left(\mathrm{IC}_{50}, 17.5 \mu \mathrm{M}\right)$ was used as a positive control [22].

Nobilin D $\left(\mathbf{2 1} ; \mathrm{IC}_{50}, 15.3 \mu \mathrm{M}\right)$, nobilin E (33; $\mathrm{IC}_{50}$, 19.2 $\mu \mathrm{M})$, nobilone $\left(\mathbf{1 2 8} ; \mathrm{IC}_{50}, 38.1 \mu \mathrm{M}\right)$, chrysotobibenzyl (16; $\left.\mathrm{IC}_{50}, 48.2 \mu \mathrm{M}\right)$, moscatilin $\left(9 ; \mathrm{IC}_{50}, 36.8 \mu \mathrm{M}\right)$, gigantol (7; $\left.\mathrm{IC}_{50}, 32.9 \mu \mathrm{M}\right)$, and dendroflorin $\left(127 ; \mathrm{IC}_{50}, 13.4 \mu \mathrm{M}\right)$ from $D$. nobile stems collected from Yunnan, China, exhibited inhibitory effects on NO production in the murine macrophage-like cell line RAW264.7 activated by LPS and IFN- $\gamma$. Resveratrol $\left(\mathrm{IC}_{50}, 23.5 \mu \mathrm{M}\right)$ was used as a positive control [23].

Ephemeranthol A (63) was isolated from D. nobile stems, and its anti-inflammatory activity was evaluated in Raw 264.7 cells. This compound reduced NO production in a dose-dependent manner. Notably, with pretreatment with $12.5 \mu \mathrm{g} / \mathrm{mL}$ ephemeranthol $\mathrm{A}$, NO production decreased to the level of the cell-only control [24]. 


\subsubsection{Dendrobium officinale Kimura \& Migo}

Vicenin 2 (vicenin II, 108) was suggested to be a common component in D. officinale leaves of different origins [25]. This di- $C$-glucosylflavone was synthesized, and it inhibited TNF- $\alpha$ expression and NO production with $\mathrm{IC}_{50}$ values of 6.8 and $3.9 \mu \mathrm{M}$, respectively, as compared with the positive control apigenin $\left(\mathrm{IC}_{50}=18.5\right.$ and $19.0 \mu \mathrm{M}$, respectively) [26].

Two types of polysaccharides in D. officinale leaves, DLP-1 and DLP-2, were obtained by hot water extraction. DLP-1 $(5 \mu \mathrm{g} / \mathrm{mL})$ and DLP-2 $(50 \mu \mathrm{g} / \mathrm{mL})$ were shown to be effective in protecting THP-1 cells, a human leukemia monocytic cell line, from LPS-stimulated cytotoxicity and inhibited reactive oxygen species formation. In addition, both DLP-1 $(5 \mu \mathrm{g} / \mathrm{mL})$ and DLP-2 $(50 \mu \mathrm{g} / \mathrm{mL})$ significantly suppressed TLR4, myeloid differentiation factor (MyD88), and tumor necrosis factor receptor-associated factor-6 (TRAF6) mRNA and protein expression in LPS-stimulated THP-1 cells [27].

\subsubsection{Dendrobium parishii H. Low}

(-)-Dendroparishiol (36) from the whole plant of D. parishii collected from Thailand was evaluated for its antiinflammatory effects in LPS-stimulated RAW264.7 murine macrophage cells. At 12.5, 25 and $50 \mu \mathrm{g} / \mathrm{mL},(-)$-dendroparishiol reduced the expression of iNOS and COX-2 in LPStreated RAW264.7 cells [28].

\subsubsection{Dendrobium cv. Sonia}

Ten compounds were isolated from Dendrobium cv. Sonia stems collected from Nanjing, China, and their anti-inflammatory activities were evaluated. 6-Feruloyloxyhexanoic ester $\left(\mathbf{1 1 2} ; \mathrm{IC}_{50}, 29.6 \mu \mathrm{M}\right)$, pinoresinol $\left(\mathbf{1 1 9} ; \mathrm{IC}_{50}, 26.3 \mu \mathrm{M}\right)$, syringaresinol $\left(\mathbf{1 2 2} ; \mathrm{IC}_{50}, 27.7 \mu \mathrm{M}\right)$, and sesqui-illisimonan $\mathrm{A}\left(\mathbf{1 2 4} ; \mathrm{IC}_{50}, 31.6 \mu \mathrm{M}\right)$ exhibited inhibitory activities on $\mathrm{NO}$ production. Aminoguanidine $\left(\mathrm{IC}_{50}, 27.3 \mu \mathrm{M}\right)$ was used as a positive control [29].

\subsubsection{Dendrobium tosaense Makino}

Via oral administration at dosages of 30,100 , and $300 \mathrm{mg} /$ $\mathrm{kg}$ for one week in an atopic dermatitis murine model, the standardized ethyl acetate extract of cultured $D$. tosaense stems protected mice from OVA/TNCB-induced skin lesions of atopic dermatitis [30].

\subsection{Antimicrobial Activity}

Microorganisms can cause skin infections, such as carbuncles, furuncles, cellulitis, impetigo, boils (Staphylococcus aureus), folliculitis (S. aureus, Pseudomonas aeruginosa), ringworm (Microsporum spp., Epidermophyton spp., and Trichophyton spp.), acne (Propionibacterium acnes), and foot odor (Brevibacterium spp.) [31].

Gigantol (7) has been found in 33 Dendrobium species [32]. This compound showed inhibitory activity against Staphylococcus aureus with an MIC value of $82.2 \mu \mathrm{g} / \mathrm{mL}$ [33].

Sortase A (srtA), a transpeptidase in gram-positive bacteria, can anchor surface proteins that play a vital role in the pathogenesis of these bacteria. SrtA is known as a potential antivirulent drug target to treat bacterial infections. Erianin (14) was isolated from $D$. chrysotoxum stems [34]. This compound could inhibit the activity of srtA in vitro with an $\mathrm{IC}_{50}$ value of $20.9 \mu \mathrm{g} / \mathrm{mL}$ [35].

Dendrocoumarin (125) and itolide A (126) from D. nobile stems collected from Hainan, China, showed antibacterial activities against Staphylococcus aureus with the same MIC value of $2.5 \mu \mathrm{g} / \mathrm{mL}$ and Micrococcus tetragenus with the same MIC value of $5.0 \mu \mathrm{g} / \mathrm{mL}$. The positive control was ciprofloxacin, with MIC values of 0.6 and $0.3 \mu \mathrm{g} / \mathrm{mL}$ for the two bacteria, respectively [36].

\subsection{Antioxidant and Antiaging Effects}

The skin shows obvious signs of aging due to age, ultraviolet radiation exposure, and chemical pollution [37]. The changes in the skin are among the most visible signs of aging, including wrinkles, sagging skin, age spots dryness, and the loss of fat, which cause the skin to lose its natural smoothness [38]. The sum of the deleterious free radical reactions is a major contributor to the aging process [39]. In intrinsically aged skin, the quantity of dermal collagen decreases, and elastin accumulates structural abnormalities [40].

\subsubsection{Dendrobium amoenum Wall. Ex Lindl.}

Chloroform and acetone extracts of D. amoenum stems collected from Nepal showed 2,2-diphenyl-1-picrylhydrazyl (DPPH) free radical scavenging activities with $\mathrm{IC}_{50}$ values of 36.5 and $53.2 \mu \mathrm{g} / \mathrm{mL}$, respectively [41].

\subsubsection{Dendrobium aphyllum (Roxb.) C.E.C. Fisch.}

Aphyllone B (17) from the stems of D. aphyllum collected from Yunnan, China, possessed DPPH radical scavenging activity with a scavenging percentage of $88 \%$ at a concentration of $100 \mu \mathrm{g} / \mathrm{mL}$ [42].

\subsubsection{Dendrobium crepidatum Lindl. \& Paxton}

Ethanol and acetone extracts of D. crepidatum stems collected from Nepal showed DPPH free radical scavenging 
activities with $\mathrm{IC}_{50}$ values of 73.9 and $99.4 \mu \mathrm{g} / \mathrm{mL}$, respectively, as compared with the positive control ascorbic acid $\left(\mathrm{IC}_{50}, 38.2 \mu \mathrm{g} / \mathrm{mL}\right)[43]$.

\subsubsection{Dendrobium denneanum Kerr}

The ethanolic extract of $D$. denneanum [syn. D. aurantiacum var. denneanum (Kerr) Z.H. Tsi] stems collected from Yunnan, China, exhibited DPPH radical scavenging activity with an $\mathrm{IC}_{50}$ value of $92.6 \mu \mathrm{g} / \mathrm{mL}$ and was compared with $\alpha$-tocopherol ( $\mathrm{IC}_{50}, 25 \mu \mathrm{g} / \mathrm{mL}$ ) as a positive control. Three compounds were obtained by bioguided isolation. Unfortunately, the activities of these compounds were weaker than those of the crude extract [44].

A bibenzyl-rich fraction from $D$. denneanum stems collected from Sichuan, China, exhibited DPPH scavenging activity with an $\mathrm{EC}_{50}$ of $62.8 \mu \mathrm{g} / \mathrm{mL}$. Vitamin $\mathrm{C}\left(\mathrm{EC}_{50}\right.$, $3.4 \mu \mathrm{g} / \mathrm{mL}$ ) was used as a positive control [45].

\subsubsection{Dendrobium denudans D. Don}

The methanol extract of $D$. denudans stems collected from India showed in vitro antioxidant activity by a reducing power assay with an $\mathrm{IC}_{50}$ value of $10.1 \mu \mathrm{g} / \mathrm{mL}$. Ascorbic acid $\left(\mathrm{IC}_{50}, 3.9 \mu \mathrm{g} / \mathrm{mL}\right)$ was used as a positive control [46].

\subsubsection{Dendrobium draconis Rchb.f.}

5-Methoxy-7-hydroxy-9,10-dihydro-1,4-phenanthrenequinone $\left(69 ; \mathrm{IC}_{50}, 283.3 \mu \mathrm{M}\right.$ or $\left.72.6 \mu \mathrm{g} / \mathrm{mL}\right)$, hircinol (55; $\left.\mathrm{IC}_{50}, 22.3 \mu \mathrm{M}\right)$, gigantol $\left(7 ; \mathrm{IC}_{50}, 17.7 \mu \mathrm{M}\right)$, and 7-methoxy9,10-dihydrophenanthrene-2,4,5-triol $\left(\mathbf{5 9} ; \mathrm{IC}_{50}, 10.2 \mu \mathrm{M}\right)$ were isolated from $D$. draconis stems collected from Thailand and exhibited DPPH radical scavenging activities. Quercetin $\left(\mathrm{IC}_{50}, 2.4 \mu \mathrm{M}\right)$ and Trolox $\left(\mathrm{IC}_{50}, 11.7 \mu \mathrm{M}\right)$ were used as positive controls [47].

\subsubsection{Dendrobium huoshanense Z.Z. Tang \& S.J. Cheng}

A polyphenol extract was obtained from $D$. huoshanense collected from Anhui, China, and the extract exhibited DPPH and 2,2'-azino-bis(3-ethylbenzothiazoline-6-sulfonic acid) diammonium salt (ABTS) radical scavenging activities with $\mathrm{IC}_{50}$ values of 57 and $27 \mu \mathrm{g} / \mathrm{mL}$, respectively, as compared with the positive control vitamin C [48].

\subsubsection{Dendrobium loddigesii Rolfe}

A series of phenolic compounds were found in D. loddigesii collected from Yunnan, China. From its stems, threo-7-O-ethyl-9-O-(4-hydroxyphenyl)propionylguaiacylglycerol (113), crepidatin (15), moscatilin (9), 4,5,4'-trihydroxy-3,3'-dimethoxybibenzyl (13), 4',5-dihydroxy-3,3'-dimethoxybibenzyl (gigantol, 7), tristin (8), dihydroconiferyl dihydro- $p$-coumarate (114), and $p$-hydroxyphenethyl trans-ferulate (115) were obtained, and these compounds exhibited significant activities, with DPPH scavenging capacities ranging from 89 to $94 \%$ at $100 \mu \mathrm{g} /$ $\mathrm{mL}$, as compared with the positive control Trolox, which led to an inhibition of $96 \%$ at a concentration of $25 \mu \mathrm{g} / \mathrm{mL}$. Batatasin III (1) significantly stimulated the collagen production activity of human dermal fibroblasts-adult (HDFa) $\left(\mathrm{EC}_{50} 3.2 \mu \mathrm{g} / \mathrm{mL}\right)$ and was compared with TGF- $\beta$ as a positive control, with an inhibition of $66 \%$ at a concentration of $0.01 \mu \mathrm{g} / \mathrm{mL}$ [49]. From complete plants, moscatin (45), 9,10-dihydrophenanthrene-2,4,7-triol (56), and crepidatuol B (27) were obtained, and these compounds showed significant activities, with DPPH scavenging capacities ranging from 84 to $95 \%$ at $100 \mu \mathrm{g} / \mathrm{mL}$, as compared with the positive control Trolox, which led to an inhibition of $96 \%$ at a concentration of $25 \mu \mathrm{g} / \mathrm{mL}$ [50].

Phenanthrenes and bibenzyls were isolated from $D$. loddigesii stems collected from Guangdong, China. Loddigesiinol A (52; $\left.\mathrm{IC}_{50}, 26.1 \mu \mathrm{M}\right)$, moscatin $\left(\mathbf{4 5} ; \mathrm{IC}_{50}, 59.8 \mu \mathrm{M}\right)$, lusianthridin $\left(\mathbf{5 4} ; \mathrm{IC}_{50}, 62.2 \mu \mathrm{M}\right)$, and loddigesiinol C (25; $\mathrm{IC}_{50}, 23.7 \mu \mathrm{M}$ ) showed activities using the DPPH-scavenging assay, as compared to the positive controls resveratrol $\left(\mathrm{IC}_{50}, 28.7 \mu \mathrm{M}\right)$ and aminoguanidine $\left(\mathrm{IC}_{50}, 21.7 \mu \mathrm{M}\right)$ [19].

Fifteen compounds were isolated from a preparation of $D$. loddigesii stems collected from Yunnan, China. Chrysotoxol A (74; $\left.\mathrm{IC}_{50}, 23.2 \mu \mathrm{M}\right), 3,6,9$-trihydroxy-3,4-dihydroanthracen-1-(2H)-one $\left(\mathbf{1 2 9} ; \mathrm{IC}_{50}, 22.8 \mu \mathrm{M}\right), 4,4^{\prime}$-dihydroxy-3,5dimethoxybibenzyl $\left(5 ; \mathrm{IC}_{50}, 94.5 \mu \mathrm{M}\right), 5,4^{\prime}$-dihydroxy7,3',5'-trimethoxyflavanone $\left(\mathbf{1 0 6} ; \mathrm{IC}_{50}, 227.7 \mu \mathrm{M}\right.$ or $78.9 \mu \mathrm{g} /$ $\mathrm{mL})$, batatasin III $\left(\mathbf{1} ; \mathrm{IC}_{50}, 204.9 \mu \mathrm{M}\right.$ or $\left.50.1 \mu \mathrm{g} / \mathrm{mL}\right), 3,3$, 5trihydroxybibenzyl $\left(2 ; \mathrm{IC}_{50}, 85.8 \mu \mathrm{M}\right)$, trigonopol B (28; $\left.\mathrm{IC}_{50}, 60.1 \mu \mathrm{M}\right)$, syringaresinol $\left(\mathbf{1 2 2} ; \mathrm{IC}_{50}, 31.1 \mu \mathrm{M}\right)$, and 9,10-dihydrophenanthrene-2,4,7-triol $\left(\mathbf{5 6} ; \mathrm{IC}_{50}, 14.1 \mu \mathrm{M}\right)$ showed DPPH radical scavenging activities. Vitamin $\mathrm{C}$ $\left(\mathrm{IC}_{50}, 29.8 \mu \mathrm{M}\right)$ was used as a positive control [20].

\subsubsection{Dendrobium longicornu Lindl.}

The acetonic extract of $D$. longicornu stems collected from Nepal showed DPPH radical scavenging activity with an $\mathrm{IC}_{50}$ value of less than $100 \mu \mathrm{g} / \mathrm{mL}$ and was compared with ascorbic acid $\left(\mathrm{IC}_{50},<50 \mu \mathrm{g} / \mathrm{mL}\right)$ as a positive control [51].

\subsubsection{Dendrobium macrostachyum Lindl.}

Ethanolic extracts of D. macrostachyum stems and leaves collected from India exhibited DPPH radical scavenging activities with $\mathrm{IC}_{50}$ values of 10.2 and $31.5 \mu \mathrm{g} / \mathrm{mL}$, respectively, as compared with the positive control ascorbic acid ( $\left.\mathrm{IC}_{50}, 18.4 \mu \mathrm{g} / \mathrm{mL}\right)$; these extracts also had ABTS radical scavenging activities with $\mathrm{IC}_{50}$ values of 31.5 and $49.1 \mu \mathrm{g} /$ 
$\mathrm{mL}$, respectively, as compared with the positive control ascorbic acid $\left(\mathrm{IC}_{50}, 34.9 \mu \mathrm{g} / \mathrm{mL}\right)$. The activities of the ethanol extracts were better than those of petroleum ether, methanol, or water extracts [52].

\subsubsection{Dendrobium moniliforme (L.) Sw.}

The DPPH radical scavenging activities of hexane, chloroform, acetone, and ethanol extracts of D. moniliforme stems collected from Nepal were measured, showing $\mathrm{IC}_{50}$ values of $52.7,42.4,49.6$, and $58.8 \mu \mathrm{g} / \mathrm{mL}$, respectively, as compared with the positive control ascorbic acid $\left(\mathrm{IC}_{50}, 38.2 \mu \mathrm{g} /\right.$ $\mathrm{mL)}$ [53].

Based on bioguided fractionation and isolation, a mixture of $n$-pentacosyl trans-ferulate (117) and $n$-tetracosyl transferulate (116) (1:4) was obtained from tissue culture-raised plants of D. moniliforme. At $100 \mu \mathrm{g} / \mathrm{mL}$, the mixture of the alkyl ferulates exhibited DPPH radical scavenging activity (inhibition`50\%) [54].

\subsubsection{Dendrobium nobile Lindl.}

Flavonoids have been detected in ethyl acetate, $n$-butanol, and aqueous extracts of $D$. nobile leaves collected from Guangdong, China. These three extracts showed DPPH free radical scavenging activities with $\mathrm{IC}_{50}$ values of 21,11 , and $13 \mu \mathrm{g} / \mathrm{mL}$, respectively, with Trolox used as a reference compound ( $\left.\mathrm{IC}_{50}, 7 \mu \mathrm{g} / \mathrm{mL}\right)$ [55].

Bibenzyls and other phenolic compounds were isolated from $D$. nobile stems collected from Yunnan, China. Nobilin A $\left(22 ; \mathrm{IC}_{50}, 87.1 \mu \mathrm{M}\right)$, nobilin B $\left(\mathbf{2 3} ; \mathrm{IC}_{50}, 32.2 \mu \mathrm{M}\right)$, nobilin $\mathrm{C}\left(\mathbf{2 4} ; \mathrm{IC}_{50}, 136.0 \mu \mathrm{M}\right.$ or $\left.47.4 \mu \mathrm{g} / \mathrm{mL}\right)$, dihydroconiferyl alcohol $\left(109 ; \mathrm{IC}_{50}, 50.9 \mu \mathrm{M}\right)$, coniferylaldehyde $\left(110 ; \mathrm{IC}_{50}\right.$, $127.9 \mu \mathrm{M}$ or $22.8 \mu \mathrm{g} / \mathrm{mL}$ ), 3-hydroxy-4-methoxyphenylethanol $\left(130 ; \mathrm{IC}_{50}, 64.5 \mu \mathrm{M}\right)$, and syringic acid $\left(131 ; \mathrm{IC}_{50}\right.$, $8.1 \mu \mathrm{M})$ exhibited DPPH radical scavenging activities. Vita$\min \mathrm{C}\left(\mathrm{IC}_{50}, 18.0 \mu \mathrm{M}\right)$ and $\mathrm{BHT}\left(\mathrm{IC}_{50}, 90.9 \mu \mathrm{M}\right)$ were used as positive controls [56].

Nobilin D $\left(\mathbf{2 1} ; \mathrm{IC}_{50}, 19.9 \mu \mathrm{M}\right)$, nobilin E $\left(\mathbf{3 3} ; \mathrm{IC}_{50}\right.$, $21.0 \mu \mathrm{M})$, crepidatin $\left(\mathbf{1 5} ; \mathrm{IC}_{50}, 21.8 \mu \mathrm{M}\right)$, dendrobin A (10; $\left.\mathrm{IC}_{50}, 40.3 \mu \mathrm{M}\right)$, chrysotoxine $\left(11 ; \mathrm{IC}_{50}, 14.0 \mu \mathrm{M}\right)$, moscatilin $\left(9 ; \mathrm{IC}_{50}, 14.5 \mu \mathrm{M}\right)$, gigantol $\left(7 ; \mathrm{IC}_{50} 56.4 \mu \mathrm{M}\right)$, and dendroflorin $\left(127 ; \mathrm{IC}_{50}, 16.2 \mu \mathrm{M}\right)$ from $D$. nobile stems exhibited DPPH radical scavenging activities. Vitamin $\mathrm{C}\left(\mathrm{IC}_{50}\right.$, $18.0 \mu \mathrm{M}$ ) was used as a positive control [23].

Phenanthrenes and lignans were isolated from $D$. nobile stems in Yunnan, China. Fimbriatone $\left(\mathbf{7 0} ; \mathrm{IC}_{50}, 144.5 \mu \mathrm{M}\right.$ or $40.8 \mu \mathrm{g} / \mathrm{mL})$, confusarin $\left(47 ; \mathrm{IC}_{50}, 12.9 \mu \mathrm{M}\right)$, flavanthrinin (49; $\left.\mathrm{IIC}_{50}, 35.7 \mu \mathrm{M}\right), 2,5$-dihydroxy-4,9-dimethoxyphenanthrene $\left(\mathbf{5 3} ; \mathrm{IC}_{50}, 34.8 \mu \mathrm{M}\right), 5,7$-dimethoxyphenanthrene2,6-diol $\left(\mathbf{5 0} ; \mathrm{IC}_{50}, 29.7 \mu \mathrm{M}\right)$, syringaresinol $\left(\mathbf{1 2 2} ; \mathrm{IC}_{50}\right.$, $9.8 \mu \mathrm{M})$, pinoresinol $\left(119 ; \mathrm{IC}_{50}, 60.6 \mu \mathrm{M}\right)$, medioresinol $\left(\mathbf{1 2 1} ; \mathrm{IC}_{50}, 27.9 \mu \mathrm{M}\right)$, and lirioresinol $\mathrm{A}\left(\mathbf{1 2 3} ; \mathrm{IC}_{50}, 30.9 \mu \mathrm{M}\right)$ exhibited DPPH radical scavenging activities. Vitamin C $\left(\mathrm{IC}_{50}, 18.0 \mu \mathrm{M}\right)$ and BHT $\left(\mathrm{IC}_{50}, 90.9 \mu \mathrm{M}\right)$ were used as positive controls. For all phenanthrenes and lignans, an electrondonating methoxy group in the ortho position that donates to the phenolic hydroxy group exhibits enhanced antioxidant activity [57].

\subsubsection{Dendrobium officinale Kimura \& Migo}

Ethanolic extracts of D. officinale flowers, leaves, and stems collected from Zhejiang, China, in which the total flavonoid contents measured were $1.8,0.25$, and $0.052 \%$, respectively, were prepared. These extracts showed DPPH scavenging activities with $\mathrm{IC}_{50}$ values of $0.2 \mu \mathrm{g} / \mathrm{mL}, 17.4 \mu \mathrm{g} / \mathrm{mL}$, and $10.4 \mu \mathrm{g} / \mathrm{mL}$, respectively, as compared with the positive control vitamin $\mathrm{C}\left(\mathrm{IC}_{50}, 7.5 \mu \mathrm{g} / \mathrm{mL}\right)$ [58].

The chloroform extract of D. officinale stems collected from Yunnan, China, exhibited ABTS radical scavenging activity with an $\mathrm{IC}_{50}$ value of $88.8 \mu \mathrm{g} / \mathrm{mL}$ [59].

The antioxidant activities of D. officinale (Syn. D. candidum) collected from different areas of Hainan, China, were compared. Ethanolic extracts of plant samples collected from Sanya, Qiongzhong, and Baoting exhibited the best DPPH radical scavenging activities, with $\mathrm{IC}_{50}$ values of 15.9, 20.2 , and $78.7 \mu \mathrm{g} / \mathrm{mL}$, respectively [60].

A series of bibenzyls were isolated from $D$. officinale (syn. D. candidum) stems collected from Zhejiang, China. Dendrocandin $\mathrm{C}\left(\mathbf{1 8} ; \mathrm{IC}_{50}, 34.2 \mu \mathrm{M}\right)$, dendrocandin $\mathrm{D}$ (19; $\left.\mathrm{IC}_{50}, 34.5 \mu \mathrm{M}\right)$, dendrocandin $\mathrm{E}\left(\mathbf{1 2} ; \mathrm{IC}_{50}, 15.6 \mu \mathrm{M}\right)$ [61], dendrocandin $\mathrm{F}\left(\mathbf{2 9} ; \mathrm{IC}_{50}, 55.8 \mu \mathrm{M}\right)$, dendrocandin $\mathrm{G}$ $\left(\mathbf{3 0} ; \mathrm{IC}_{50}, 32.4 \mu \mathrm{M}\right)$, dendrocandin $\mathrm{H}\left(\mathbf{3 4} ; \mathrm{IC}_{50}, 19.8 \mu \mathrm{M}\right)$, dendrocandin $\mathrm{I}\left(\mathbf{3 9} ; \mathrm{IC}_{50}, 21.3 \mu \mathrm{M}\right)[62]$, dendrocandin $\mathrm{J}$ (31; $\left.\mathrm{IC}_{50}, 36.8 \mu \mathrm{M}\right)$, dendrocandin $\mathrm{K}\left(\mathbf{3 2} ; \mathrm{IC}_{50}, 70.2 \mu \mathrm{M}\right)$, dendrocandin $\mathrm{L}\left(\mathbf{3 5} ; \mathrm{IC}_{50}, 45.0 \mu \mathrm{M}\right)$, dendrocandin $\mathrm{M}(\mathbf{3 7}$; $\left.\mathrm{IC}_{50}, 60.5 \mu \mathrm{M}\right)$, dendrocandin $\mathrm{N}\left(\mathbf{4 2} ; \mathrm{IC}_{50}, 87.6 \mu \mathrm{M}\right)$, dendrocandin $\mathrm{O}\left(\mathbf{4 3} ; \mathrm{IC}_{50}, 50.4 \mu \mathrm{M}\right)$, dendrocandin $\mathrm{P}\left(\mathbf{4 0} ; \mathrm{IC}_{50}\right.$, $22.3 \mu \mathrm{M})$, dendrocandin $\mathrm{Q}\left(\mathbf{4 1} ; \mathrm{IC}_{50}, 30.3 \mu \mathrm{M}\right)$ [63], and (S)-3,4, $\alpha$-trihydroxy-5,4'-dimethoxybibenzyl $\left(\mathbf{2 0} ; \mathrm{IC}_{50}, \mathrm{IC}_{50}\right.$, $32.3 \mu \mathrm{M})$ exhibited DPPH radical scavenging activities [64]. Vitamin $\mathrm{C}\left(\mathrm{IC}_{50}, 23.2 \mu \mathrm{M}\right)$ was used as a positive control [61-64].

Moupinamide (100; $\left.\mathrm{IC}_{50}, 53.8 \mu \mathrm{M}\right)$, dihydroconiferyl dihydro-p-cumarate $\left(\mathbf{1 1 4} ; \mathrm{IC}_{50}, 78.2 \mu \mathrm{M}\right)$, dihydroferuloyltyramine $\left(\mathbf{1 0 1} ; \mathrm{IC}_{50}, 113.5 \mu \mathrm{M}\right.$ or $\left.35.8 \mu \mathrm{g} / \mathrm{mL}\right)$, syringic acid $\left(131 ; \mathrm{IC}_{50}, 36.5 \mu \mathrm{M}\right)$, ferulic acid $\left(111 ; \mathrm{IC}_{50}, 64.9 \mu \mathrm{M}\right)$, and 3',5,5',7-tetrahydroxyflavanone $\left(\mathbf{1 0 5} ; \mathrm{IC}_{50}, 29.8 \mu \mathrm{M}\right)$ from $D$. officinale (syn. D. catenatum) stems collected from Zhejiang, China, showed DPPH free radical scavenging activities. Vitamin $\mathrm{C}\left(\mathrm{IC}_{50}, 23.2 \mu \mathrm{M}\right)$ was used as a positive control [65].

Eight bibenzyls were isolated from $D$. officinale (syn. $D$. catenatum) stems collected from Shenzhen, China. Dendrocandin U (44), 3,4'-dihydroxy-5-methoxybibenzyl (3), and 
3,4',5-trihydroxy-3'-methoxybibenzyl (tristin, 8) exhibited significant ABTS radical scavenging activities with $\mathrm{IC}_{50}$ values of 10.0, 5.3, and $9.0 \mu \mathrm{M}$, respectively, as compared with the positive control vitamin $\mathrm{C}\left(\mathrm{IC}_{50}, 6.5 \mu \mathrm{M}\right) .3,4^{\prime}, 5-$ Trihydroxy-3'-methoxybibenzyl also exhibited DPPH scavenging activity with an $\mathrm{IC}_{50}$ value of $34.5 \mu \mathrm{M}$. Vitamin $\mathrm{C}$ $\left(\mathrm{IC}_{50}, 14.9 \mu \mathrm{M}\right)$ was used as a positive control [66].

D. officinale protocorm powder in deionized water (10, 25 and $50 \mathrm{mg} / \mathrm{mL}$, external administration) significantly reduced erythema and protected the skin from dryness in a hairless mouse model with UV irradiation-induced skin damage using matrixyl $(10 \mathrm{mg} / \mathrm{mL})$ as a positive control. This study demonstrated that $D$. officinale protocorms can inhibit photodamage in the skin of hairless mice [67].

An in vivo experiment using photoaged model mice was conducted. The results showed that the ultrafine powder and fine powder of $D$. officinale (syn. $D$. candidum) stems possess a certain preventive effect on photoaging, and the effect of ultrafine powder is better than that of fine powder [68].

\subsubsection{Dendrobium palpebrae Lindl.}

A fluorenone, dendroflorin (127), was isolated from $D$. palpebrae whole plants collected from Thailand. This compound significantly decreased ROS in $\mathrm{H}_{2} \mathrm{O}_{2}$-stimulated RAW264.7 cells in a dose-dependent manner $(12.5-50 \mu \mathrm{g} /$ $\mathrm{mL)}[69]$.

\subsubsection{Dendrobium secundum (Blume) Lindl. ex Wall.}

Five compounds were isolated from $D$. secundum stems collected from Thailand. 4,5,4'-Trihydroxy-3,3'dimethoxybibenzyl $\left(\mathbf{1 3} ; \mathrm{IC}_{50} 15.9 \mu \mathrm{M}\right)$, moscatilin $\left(\mathbf{9} ; \mathrm{IC}_{50}\right.$ $5.1 \mu \mathrm{M})$, syringaresinol $\left(122 ; \mathrm{IC}_{50} 11.4 \mu \mathrm{M}\right)$, and ferulic acid (111; $\left.\mathrm{IC}_{50} 37.5 \mu \mathrm{M}\right)$ exhibited DPPH free radical scavenging activities, which were compared with the positive controls quercetin and Trolox $\left(\mathrm{IC}_{50}, 2.5\right.$ and $11.7 \mu \mathrm{M}$, respectively) [70].

\subsubsection{Dendrobium signatum Rchb.f.}

D. signatum leaves collected from Thailand were extracted with ethanol by sonication-maceration for $30 \mathrm{~min}$. The extract showed DPPH radical scavenging activity, with a measured $\mathrm{IC}_{50}$ value of $97.2 \mu \mathrm{g} / \mathrm{mL}$, with ascorbic acid used as a reference compound $\left(\mathrm{IC}_{50}, 21.7 \mu \mathrm{g} / \mathrm{mL}\right)[71]$.

\subsubsection{Dendrobium speciosum Sm.}

Methanolic extract of $D$. speciosum leaves collected from Australia containing polyphenols $(1.2 \%)$ and flavonoids $(0.2 \%)$ showed DPPH scavenging activity, with a measured $\mathrm{IC}_{50}$ value of $26 \mu \mathrm{g} / \mathrm{mL}$. Trolox was used as a reference compound $\left(\mathrm{IC}_{50}\right.$, $20 \mu \mathrm{g} / \mathrm{mL})$ [72].

\subsubsection{Dendrobium tosaense Makino}

The effects of methanolic extracts obtained from three Dendrobium species propagated in vitro on DPPH scavenging were investigated. The $D$. tosaense extract was the most active extract, with an $\mathrm{IC}_{50}$ value of $79.9 \mu \mathrm{g} / \mathrm{mL}$, as compared with the positive control $\alpha$-tocopherol $\left(\mathrm{IC}_{50}, 58.2 \mu \mathrm{M}\right)$ [73].

Based on bioguided fractionation and isolation, quercetin (103) was obtained from tissue culture-raised plants of $D$. tosaense. At $100 \mu \mathrm{g} / \mathrm{mL}$, quercetin exhibited DPPH radical scavenging activity (inhibition > 50\%) [54].

\subsubsection{Dendrobium williamsonii Day \& Rchb.f.}

Six compounds were isolated from $D$. williamsonii whole plants collected from Thailand and evaluated these isolates for their DPPH radical scavenging activities. 3,3'-Dihydroxy-4,5-dimethoxybibenzyl (6), moscatilin (9), and apigenin (102) were active, with $\mathrm{IC}_{50}$ values of 19.5, 8.5 , and $19.3 \mu \mathrm{M}$, respectively, as compared with the positive controls, quercetin $\left(\mathrm{IC}_{50}, 8.3 \mu \mathrm{M}\right)$ and vitamin $\mathrm{C}\left(\mathrm{IC}_{50}\right.$ $42.4 \mu \mathrm{M})$ [74].

\subsection{Anti-Psoriasis Activity}

Psoriasis is a recurrent skin disease described as keratinocyte hyperproliferation and aberrant differentiation. At concentrations ranging from $12.5 \mathrm{nM}$ to $50 \mathrm{nM}$, erianin (14) inhibited proliferation and induced apoptosis in a human keratinocyte cell line ( $\mathrm{HaCaT})$. Erianin could be recognized as a potential anti-psoriasis drug [75]. This compound was previously isolated from $D$. chrysotoxum [34].

\subsection{Hair Growth Promoting Effects}

Alopecia is a skin disease characterized by reduced hair [76]. The condition has a strong influence on the mental and psychological health of patients [77].

In an in vivo experiment, C57BL/6 J mice were externally administered $D$. officinale (Guangdong, China) polysaccharides (DOP, $5.0 \mathrm{~g} / \mathrm{L}$ ) for 21 days. The average hair growth score and average quality of $\mathrm{C} 57 \mathrm{BL} / 6 \mathrm{~J}$ mice in the DOP group were significantly better than those in the control groups [78].

\subsection{Skin-Moisturizing Effects}

When the water content in the stratum corneum drops to less than $10 \%$, the skin appears dry, loses elasticity, and wrinkles and skin aging accelerates [77]. 
The moisture retention rate of $D$. huoshanense (Anhui, China) polysaccharide on human skin is significantly higher than that of glycerol after external administration for $6 \mathrm{~h}, 8 \mathrm{~h}$ and $12 \mathrm{~h}$. The stimulus value of normal skin and damaged skin of rabbits is less than 0.5 , indicating that $D$. huoshanense polysaccharides do not cause skin irritation [79].

Polysaccharides from white orchids (Dendrobium cv. Khao Sanan) flowers cultivated in Thailand were evaluated in vivo for their skin hydration efficacy in human volunteers. The efficacy of white orchid polysaccharides at $0.3 \%$ was noted to be superior in terms of skin hydration efficacy than sea weed polysaccharides at $0.2 \%$ [80].

An in vivo experiment was conducted to evaluate the moisturizing effects of a $D$. nobile stem extract on human skin. The moisturizing abilities after $30 \mathrm{~min}$ and $2 \mathrm{~h}$ were greater than 1 for the D. nobile stem extract (2.0\%) [81].

An in vivo experiment showed that $20 \mu \mathrm{g} / \mathrm{mL}$ ethanolic extract of D. officinale collected from Yunnan, China, exhibited a skin moisturizing effect. After $2.5 \mathrm{~h}$ of use, skin hydrature increased by $16 \%$ compared with that before use $(P<0.05)[82]$.

\subsection{Tyrosinase-Inhibitory Activity}

Tyrosinase inhibitors are used for hyperpigmentation and developing skin whitening agents.

3,3',5-Trihydroxybibenzyl (2) from D. loddigesii stems collected from Yunnan, China, revealed significant inhibitory activity against tyrosinase with an $\mathrm{IC}_{50}$ value of $37.9 \mu \mathrm{g} /$ $\mathrm{mL}$, as compared with the positive control kojic acid $\left(\mathrm{IC}_{50}\right.$, $8.0 \mu \mathrm{g} / \mathrm{mL}$ ) [49].

At concentrations of $12.5,25$ and $50 \mu \mathrm{g} / \mathrm{mL}$, the $\mathrm{CH}_{2} \mathrm{Cl}_{2}$ extract of D. moniliforme leaves collected from South Korea inhibited melanogenesis in murine melanoma cells (B16F10), implying that D. moniliforme is effective against hyperpigmentation disorders and that it is considered a possible antimelanogenic agent in topical application [83].

Ethanolic extracts of Dendrobium cv. Sonia flowers and Dendrobium cv. Sonia pink flowers collected from Thailand showed tyrosinase inhibitory activities using L-tyrosine as a substrate. The $\mathrm{IC}_{50}$ values were $57.4 \mu \mathrm{g} / \mathrm{mL}$ and $83.2 \mu \mathrm{g} /$ $\mathrm{mL}$, respectively, as compared with the positive control kojic acid $\left(\mathrm{IC}_{50}, 151.7 \mu \mathrm{g} / \mathrm{mL}\right)$ [84].

\section{Conclusion}

There are 22 Dendrobium species with traditional uses for treating dermatological disorders by local people in eight countries, and there are 131 compounds from Dendrobium plants reported to possess anti-inflammatory, antimicrobial, antioxidant, antiaging, anti-psoriasis, and tyrosinase-inhibitory activities, which implies that Dendrobium plants are important resources for the discovery of active compounds and the development of new drugs and cosmetics. However, only $D$. crepidatum, $D$. denneanum, $D$. loddigesii, $D$. nobile, and $D$. officinale have been extensively studied. More research on other Dendrobium species is needed.

The major active compounds found in Dendrobium species are phenanthrenes, alkaloids, flavonoids, phenylpropanoids, and lignans. Several compounds, such as loddigesiinol A, (S)-5-methoxy-2,4,7,9-tetrahydroxy-9,10-dihydrophenanthrene, $(S)$-4-methoxy-2,5,7,9-tetrahydroxy-9,10-dihydrophenanthrene, 2,5-dihydroxy-4-methoxy-phenanthrene 2 - $O$ - $\beta$-D-glucopyranoside, (9R)-1,2,5,9-tetrahydroxy9,10-dihydrophenanthrene 5- $O$ - $\beta$-D-glucopyranoside, (+)-homocrepidine A, and vicenin 2 , have significant antiinflammatory activities and inhibit $\mathrm{NO}$ production with $\mathrm{IC}_{50}$ values less than $5 \mu \mathrm{M}$, and these compounds are worthy of further study.

Acknowledgments This research was funded by the Beijing DR PLANT Biotechnology Co., Ltd. and the Second Tibetan Plateau Scientific Expedition and Research (STEP) Program of Ministry of Science and Technology of the People's Republic of China (2019QZKK0502).

\section{Declarations}

Conflict of interest The author declares that there are no conflicts of interest associated with this work.

Open Access This article is licensed under a Creative Commons Attribution 4.0 International License, which permits use, sharing, adaptation, distribution and reproduction in any medium or format, as long as you give appropriate credit to the original author(s) and the source, provide a link to the Creative Commons licence, and indicate if changes were made. The images or other third party material in this article are included in the article's Creative Commons licence, unless indicated otherwise in a credit line to the material. If material is not included in the article's Creative Commons licence and your intended use is not permitted by statutory regulation or exceeds the permitted use, you will need to obtain permission directly from the copyright holder. To view a copy of this licence, visit http://creativecommons.org/licenses/by/4.0/.

\section{References}

1. R.D. Sontheimer, J. Investig. Dermatol. 134, 581-582 (2014)

2. V.V. Wagh, A.K. Jain, J. Herb. Med. 19, 100234 (2020)

3. P. Bhattacharyya, P. Paul, S. Kumaria, P. Tandon, Acta Physiol. Plant. 40, 137 (2018)

4. S.-G. Zheng, Y.-D. Hu, R.-X. Zhao, S. Yan, X.-Q. Zhang, T.-M. Zhao, Z. Chun, Planta 248, 769-784 (2018)

5. Plants of the World online, http://www.plantsoftheworldonline. org/taxon/urn:lsid:ipni.org:names:325886-2. Accessed on March 4, 2021.

6. CIRS, Inventory of Existing Chemical Ingredient in China. http:// www.cirs-reach.com/news-and-articles/new-inventory-of-exist ing-cosmetic-ingredients-in-china-launched-(iecic-2015,-finalversion).html. Accessed on March 4, 2021. 
7. E.S. Teoh, Orchids as Aphrodisiac, Medicine Or Food (Springer, Singapore, 2019), pp. 79-282

8. H.S. Lim, S.R. Yo, M.Y. Lee, C.S. Seo, H.K. Shin, S.J. Jeong, Mol. Med. Rep. 17, 2515-2522 (2018)

9. L. Yang, L.H. Qin, S.A. Bligh, A. Bashall, C.F. Zhang, M. Zhang, Z.T. Wang, L.S. Xu, Bioorg. Med. Chem. 14, 3496-3501 (2006)

10. Y. Hu, J. Ren, L. Wang, X. Zhao, M. Zhang, K. Shimizu, C. Zhang, Phytochemistry 149, 12-23 (2018)

11. Y. Hu, C. Zhang, X. Zhao, Y. Wang, D. Feng, M. Zhang, H. Xie, J. Nat. Prod. 79, 252-256 (2016)

12. Y. Hu, H. Yang, X. Ding, J. Liu, X. Wang, L. Hu, M. Liu, C. Zhang, Bioorg. Chem. 100, 103809 (2020)

13. Y. Lin, F. Wang, L.J. Yang, Z. Chun, J.K. Bao, G.L. Zhang, Phytochemistry 95, 242-251 (2013)

14. D. Yang, Z.Q. Cheng, L. Yang, B. Hou, J. Yang, X.N. Li, C.T. Zi, F.W. Dong, Z.H. Liu, J. Zhou, Z.T. Ding, J.M. Hu, J. Nat. Prod. 81, 227-235 (2018)

15. X.-B. Yang, S. Yan, J.-M. Hu, J. Zhou, Nat. Prod. Res. Dev. 31, 1745-1752 (2019)

16. Editorial Board of Chinese Pharmacopoeia, Chinese Pharmacopoeia (China Medical Science Press, Beijing, 2020), pp. 94-97

17. K.G. Wu, T.H. Li, C.J. Chen, H.I. Cheng, T.Y. Wang, Int. J. Immunopathol. Pharmacol. 24, 367-375 (2011)

18. H. Chen, X. Li, Y. Xu, K. Lo, H. Zheng, H. Hu, J. Wang, Y. Lin, Molecules 23, 1185 (2018)

19. M. Ito, K. Matsuzaki, J. Wang, A. Daikonya, N.L. Wang, X.S. Yao, S. Kitanaka, Chem. Pharm. Bull. 58, 628-633 (2010)

20. X. Li, H. Chen, W. He, W. Yang, F. Ni, Z. Huang, H. Hu, J. Wang, Acta Sci. Nat. Univ. Sunyatseni 58, 96-102 (2019)

21. T.H. Lin, S.J. Chang, C.C. Chen, J.P. Wang, L.T. Tsao, J. Nat. Prod. 64, 1084-1086 (2001)

22. J.S. Hwang, S.A. Lee, S.S. Hong, X.H. Han, C. Lee, S.J. Kang, D. Lee, Y. Kim, J.T. Hong, M.K. Lee, B.Y. Hwang, Bioorg. Med. Chem. Lett. 20, 3785-3787 (2010)

23. X. Zhang, J.K. Xu, J. Wang, N.L. Wang, H. Kurihara, S. Kitanaka, X.S. Yao, J. Nat. Prod. 70, 24-28 (2007)

24. J.H. Kim, S.-Y. Oh, S.-B. Han, G.M. Uddin, C.Y. Kim, J.K. Lee, Arch. Pharm. Res. 38, 1117-1126 (2015)

25. X.F. Zhang, C.H. Zhou, L.K. Zhang, M. Jiang, Z.S. Xie, Y. Yuan, Y.C. Huang, Y.Y. Luo, G. Wei, Chin. J. Exp. Tradit. Med. Formulae 25, 29-34 (2019)

26. J.J. Shie, C.A. Chen, C.C. Lin, A.F. Ku, T.J.R. Cheng, J.M. Fang, C.H. Wong, Org. Biomol. Chem. 8, 4451-4462 (2010)

27. M. Zhang, J. Wu, J. Han, H. Shu, K. Liu, Chem. Cent. J. 12, 109 (2018)

28. V. Kongkatitham, C. Muangnoi, N. Kyokong, W. Thaweesest, K. Likhitwitayawuid, P. Rojsitthisak, B. Sritularak, Phytochem. Lett. 24, 31-38 (2018)

29. B.X. Cai, L.X. Song, H.J. Hu, Z.Z. Han, Y. Zhou, Z.T. Wang, L. Yang, Nat. Prod. Res. (2020). https://doi.org/10.1080/14786 419.2020.1782404

30. C.T. Wu, K.S. Huang, C.H. Yang, Y.C. Chen, J.W. Liao, C.L. Kuo, C.L. Chen, S.F. Lo, C.C. Hsieh, H.S. Tsay, Int. J. Pharm. 463, 193-200 (2014)

31. A. Orchard, S. van Vuuren, Evid. Based Complement. Alternat. Med. 2017, 4517971 (2017)

32. L. He, Q. Su, L. Bai, M. Li, J. Liu, X. Liu, C. Zhang, Z. Jiang, J. He, J. Shi, S. Huang, L. Guo, Eur. J. Med. Chem. 204 $112530(2020)$

33. J. Ren, X.P. Qian, Y.G. Guo, T. Li, S.K. Yan, H.Z. Jin, W.D. Zhang, Phytochem. Lett. 18, 64-67 (2016)

34. F.W. Dong, H.R. Luo, Q.L. Wan, F.Q. Xu, W.W. Fan, K.J. Wang, N. Li, J.M. Hu, Bull. Korean Chem. Soc. 33, 2247-2250 (2012)
35. P. Ouyang, X. He, Z.W. Yuan, Z.Q. Yin, H. Fu, J. Lin, C. He, X. Liang, C. Lv, G. Shu, Z.X. Yuan, X. Song, L. Li, L. Yin, Toxins 10, 385 (2018)

36. X.M. Zhou, B. Zhang, G.Y. Chen, C.R. Han, K.C. Jiang, M.Y Luo, B.Z. Meng, W.X. Li, S.D. Lin, Nat. Prod. Res. 32, 2464 2467 (2018)

37. C. Cao, Z. Xiao, Y. Wu, C. Ge, Nutrients 12, 870 (2020)

38. S. Shanbhag, A. Nayak, R. Narayan, U.Y. Nayak, Adv. Pharm. Bull. 9, 348-359 (2019)

39. D. Harman, Proc. Natl. Acad. Sci. USA 78, 7124-7128 (1981)

40. E. Russell-Goldman, G.F. Murphy, Am. J. Pathol. 190, 1356$1369(2020)$

41. M.R. Poudel, M.B. Chand, N. Karki, B. Pant, Bot. Orient. J. Plant Sci. 9, 20-26 (2015)

42. D. Yang, L.Y. Liu, Z.Q. Cheng, F.Q. Xu, W.W. Fan, C.T. Zi, F.W. Dong, J. Zhou, Z.T. Ding, J.M. Hu, Fitoterapia 100, 11-18 (2015)

43. M.R. Paudel, M.B. Chand, B. Pant, B. Pant, Biomolecules 9, 478 (2019)

44. L. Yang, H. Han, N. Nakamura, M. Hattori, Z. Wang, L. Xu, Phytother. Res. 21, 696-698 (2007)

45. F. Jia, H.L. Xia, Z.J. Ning, P. Huang, X.Y. Huang, J.J. Tang, T.M Zhang, Sci. Technol. Food Ind. 35, 62-66 (2014)

46. C.B. Singh, M.C. Devi, D.S. Thokchom, M. Sengupta, A.K Singh, J. Pharmacogn. Phytochem. 4, 6-11 (2015)

47. B. Sritularak, M. Anuwat, K. Likhitwitayawuid, J. Asian Nat Prod. Res. 13, 251-255 (2011)

48. M. Wei, Y.Y. Liu, W.R. Cai, S.H. Qian, K. Zhang, Food Mach. 32, 136-140 (2016)

49. R.J. Ma, L. Yang, X. Bai, J.Y. Li, M.Y. Yuan, Y.Q. Wang, Y. Xie, J.M. Hu, J. Zhou, Nat. Prod. Bioprospec. 9, 329-336 (2019)

50. S. Yan, R.J. Ma, L. Yang, J.Y. Li, X.B. Yang, J.M. Hu, Nat. Prod. Res. Dev. 31, 615-620 (2019)

51. M.R. Paudel, M.B. Chand, B. Pant, B. Pant, Pharmacogn. J. 9 , 499-503 (2017)

52. N.P. Sukumaran, R.H. Yadav, Anc. Sci. Life 35, 240-244 (2016)

53. M.R. Paudel, M.B. Chand, B. Pant, B. Pant, B.M.C. Complement, Altern. Med. 18, 134 (2018)

54. S.F. Lo, V. Mulabagal, C.L. Chen, C.L. Kuo, H.S. Tsay, J. Agric. Food Chem. 52, 6916-6919 (2004)

55. Y. Li, H. Li, X. Ji, Z. Cen, J. Yan, J. Wu, China Pharm. 29, 330-333 (2018)

56. X. Zhang, J.K. Xu, N.L. Wang, H. Kurihara, X.S. Yao, Z. Wang, Chin. Pharm. J. 43, 829-832 (2008)

57. X. Zhang, J.K. Xu, N.L. Wang, H. Kurihara, X.S. Yao, J. Chin. Pharm. Sci. 17, 314-318 (2008)

58. F. Li, Y. Wei, Y. Chen, Acta Chin. Med. 34, 1020-1023 (2019)

59. Q. Huang, Y. Shen, C. Zhang, A. Luo, Y. Fan, Chin. J. Appl. Environ. Biol. 20, 438-442 (2014)

60. J. Wang, H.X. Chen, L.S. Xing, Y.W. Wang, Y.H. Huang, H.Q. Liu, Nat. Prod. Res. Dev. 27, 768-773 (2015)

61. Y. Li, C.L. Wang, Y.J. Wang, S.X. Guo, J.S. Yang, X.M. Chen, P.G. Xiao, Chem. Pharm. Bull. 57, 218-219 (2009)

62. Y. Li, C.L. Wang, Y.J. Wang, F.F. Wang, S.X. Guo, J.S. Yang, P.G. Xiao, Chem. Pharm. Bull. 57, 997-999 (2009)

63. Y. Li, C.L. Wang, H.J. Zhao, S.X. Guo, J. Asian Nat. Prod. Res. 16, 1035-1043 (2014)

64. Y. Li, C.L. Wang, H.J. Zhao, S.X. Guo, Chem. Nat. Compd. 51, 1052-1054 (2015)

65. Y. Li, C.L. Wang, F.F. Wang, H.L. Dong, S.X. Guo, J.S. Yang, P.G. Xiao, Chin. Pharm. J. 45, 975-979 (2010)

66. L.J. Zhu, M.Q. Wang, Y. Qin, M.N. Wang, G.Q. Zhang, L.T. Niu, J.B. Chen, X. Zhang, X.S. Yao, J. Asian Nat. Prod. Res. (2020) https://doi.org/10.1080/10286020.2020.1826937

67. Y. Mai, Z. Niu, W. He, X. Lai, S. Huang, X. Zheng, Biol. Pharm. Bull. 42, 728-735 (2019) 
68. H. Li, L.Q. Qian, X. Chen, N.Y. Zhang, S.S. Lei, B. Li, G.Y. Lyu, S.H. Chen, China J. Chin. Mater. Med. 44, 4677-4684 (2019)

69. N. Kyokong, C. Muangnoi, W. Thaweesest, V. Kongkatitham, K. Likhitwitayawuid, P. Rojsitthisak, B. Sritularak, J. Asian Nat. Prod. Res. 21, 391-397 (2019)

70. B. Sritularak, N. Duangrak, K. Likhitwitayawuid, Z. Naturforsch. 66, 205-208 (2011)

71. T. Chimsook, In Phytochemical screening, total phenolic content, antioxidant activities and cytotoxicity of Dendrobium signatum leaves (MATEC Web of Conferences, EDP Sciences, 2016), p. 03005

72. M. Moretti, L. Cossignani, F. Messina, L. Dominici, M. Villarini, M. Curini, M.C. Marcotullio, Food Chem. 140, 660-665 (2013)

73. S.F. Lo, S.M. Nalawade, V. Mulabagal, S. Matthew, C.L. Chen, C.L. Kuo, H.S. Tsay, Biol. Pharm. Bull. 27, 731-735 (2004)

74. P. Rungwichaniwat, B. Sritularak, K. Likhitwitayawuid, Pharmacogn. J. 6, 36-41 (2014)

75. C. Mo, D. Shetti, K. Wei, Molecules 24, 2727 (2019)

76. M. Mingsan, P. Mengfan, L. Dandan, Z. Zhengwang, J. King Saud Univ. Sci. 32, 2669-2674 (2020)

77. L. Guo, J. Qi, D. Du, Y. Liu, X. Jiang, Pharm. Biol. 58, 664-673 (2020)

78. J. Chen, H. Qi, J.B. Li, Y.Q. Yi, D. Chen, X.H. Hu, M.L. Wang, X.L. Sun, X.Y. Wei, China J. Chin. Mater. Med. 39, 291-295 (2014)

79. F.L. Gu, X.P. Jiang, Y.J. Chen, B.X. Han, N.F. Chen, C.B. Wei, Nat. Prod. Res. Dev. 30, 1701-1705 (2018)

80. M. Kanlayavattanakul, T. Pawakongbun, N. Lourith, Chin. Herb. Med. 11, 400-405 (2019)

81. Y.H. Gui, X. Meng, Q.M. Liang, S.Z. Gong, Deterg. Cosmet. 40(22-24), 26 (2017)

82. M. Chen, Y. Sun, Y. Zhao, Acta Univ. Tradit. Med. Sin. Pharmacol. Shanghai 29, 70-73 (2015)

83. Y.J. Ko, S.K. Yang, S.M. Song, W.J. Yoon, K.H. Bae, J. Biol. Act. Prod. Nat. 5, 12-17 (2015)

84. A. Athipornchai, N. Jullapo, S. Afr, J. Bot. 119, 188-192 (2018)

85. N.M. Smith, J. Adelaide Bot. Gard. 14, 1-65 (1991)

86. J. Tucci, S. Wilkens, Aust. J. Rural Health 24, 156-169 (2016)

87. D. Behera, C.C. Rath, U. Mohapatra, Floric Ornam. Biotechnol. 7, 53-59 (2013)

88. U. Barua, D.K. Hore, R.S. Rathi, G. Das, Environ. Ecol. 24, 736-742 (2006)

89. R.K. Maikhuri, P.S. Ramakrishnan, J. Econ. Tax. Bot. 10, 61-78 (1992)

90. A. Subedi, B. Kunwar, Y. Choi, Y. Dai, T. van Andel, R.P. Chaudhary, H.J. de Boer, B. Gravendeel, J. Ethnobiol. Ethnomed. 9, 64 (2013)

91. M. Akhter, M.M. Hoque, M. Rahman, M.K. Huda, J. Med. Plants Stud. 5, 265-268 (2017)

92. W.H. Li, Medical records, In Compilation Committee of Local Records, in Records of Yunnan Province. (Yunnan People's Publishing House, Kunming, 1995), pp. 960-962

\section{Authors and Affiliations}

\section{Yue-Hu Wang ${ }^{1}$ (]}

Yue-Hu Wang

wangyuehu@mail.kib.ac.cn

1 Key Laboratory of Economic Plants and Biotechnology, The Yunnan Key Laboratory for Wild Plant Resources, and Bio-Innovation Center of DR PLANT, Kunming
93. H.Y. Zhang, Z.Y. Zhang, Handbook of Traditional Chinese Medicine Resources in China (Science Press, Beijing, 1994), pp. $1531-1536$

94. M. Rahamtulla, U.C. Pradhan, A.K. Roy, V. Rampilla, S.M. Khasim, Ethnomedicinal aspects of some orchids from Darjeeling Himalaya, India, in Orchid Biology: Recent Trends \& Challenges. ed. by S.M. Khasim, S.N. Hegde, M.T. González-Arnao, K. Thammasiri (Springer, Singapore, 2020), pp. 451-472

95. H. Li, Orchidaceae, in Flora Yunnanica. ed. by Z. Wu (Science Press, Beijing, 2003), pp. 100-819

96. R. Laha, P.C. Lalremruata, R. Vanlalpeka, Int. J. Basic Appl. Res. 8, 371-384 (2018)

97. L.J. Lawler, M. Slaytor, Med. J. Australia 2, 1259-1261 (1970)

98. K. Chowlu, K.S. Mahar, A.K. Das, Indian J. Nat. Prod. Resour. 8, 89-93 (2017)

99. Yunnan Medicinal Materials Company Limited, List of Traditional Chinese Medicine Resources in Yunnan (Science Press, Beijing, China, 1993), pp. 669-672

100. A.P. Tiwari, B. Joshi, A.A. Ansari, Nat. Sci. 10, 33-37 (2012)

101. P.K. Dash, S. Sahoo, S. Bal, Ethnobot. Leaflets 12, 70-78 (2008)

102. S. Jagathes Kumar, R. Ashok Kumar, G. Uma, B. Subbaiyan, V. Aravindhan, V. Balasubramaniam, Int. J. Recent Adv. Multidiscip. Res. 2, 1047-1055 (2015)

103. B. Vaidya, M. Shrestha, N. Joshee, In Report on Nepalese orchid species with medicinal properties, In Proceedings of Nepal-Japan Joint Symposium-2000, Kathmandu, Nepal (The Himalayan plants, can they save us? Proceeding of Nepal-Japan joint symposium on conservation and utilization of Himalayan medicinal resources, 2000), p. 146-152

104. M.M. Hossain, Med. Aromat. Plant Sci. Biotechnol. 3, 100-106 (2009)

105. A.R. Roy, R.S. Patel, V.V. Patel, D.S. Yadav, J. Orchid Soc. India 21, 15-17 (2007)

106. C.R. Deb, M.S. Deb, N.S. Jamir, T. Imchen, Pleione 3, 209-211 (2009)

107. R.P. Medhi, S. Chakrabarti, Indian J. Tradit. Knowl. 8, 11-16 (2009)

108. R. Yonzone, D. Lama, R.B. Bhujel, S. Rai, Indian Forester 140, 413-418 (2014)

109. A.E. Shanavaskhan, M. Sivadasan, A.H. Alfarhan, J. Thomas, Indian J. Tradit. Knowl. 11, 250-258 (2012)

110. Z.Y. Zhu, Q.H. Wei, L. Gao, The Annals of National Medicine in Yunnan (The Nationalities Publishing House of Yunnan, Kunming, 2012), pp. 186-187

111. S. Nurfadilah, IOP Conf. Ser. Earth Environ. Sci. 473, 012063 (2020)

112. J.T. Kpadehyea, E.S. Fernando, C.E. Tinio, I.E. Buot, Electron. J. Biol. 11, 165-175 (2015)

Institute of Botany, Chinese Academy of Sciences, Kunming, Yunnan 650201, People's Republic of China 